\title{
A Review of the Toxicity of Biomass Pyrolysis Liquids Formed at Low Temperatures
}

\author{
James P. Diebold
}

Thermalchemie, Inc.

57 Yank Way

Lakewood, CO 80228

National Renewable Energy Laboratory 1617 Cole Boulevard

Golden, Colorado 80401-3393

A national laboratory of the U.S. Department of Energy Operated by Midwest Research Institute for the U.S. Department of Energy

Under Contract No. DE-AC36-83CH10093

Prepared under Task No. BP613432

April 1997 
This publication was reproduced from the best available camera-ready copy submitted by the subcontractor and received no editorial review at NREL.

\section{NOTICE.}

This report was prepared as an account of work sponsored by an agency of the United States govemment. Neither the United States government nor any agency thereof, nor any of their employees, makes any warranty, express or implied, or assumes any legal liability or responsibility for the accuracy, completeness: or usefulness of any information, apparatus, product, or process disclosed, or represents that its use would not infringe privately owned rights. Reference herein to any specific commercial product, process, or service by trade name, trademark, manufacturer, or otherwise does not necessarily constitute or imply its endorsement, recommendation, or favoring by the United States govemment or any agency thereof. The views and opinions of authors expressed herein do not necessarily state or reflect those of the United States govemment or any agency thereof.

Available to DOE and DOE contractors from:

Office of Scientific and Technical Information (OSTI)

P.O. Box 62

Oak Ridge, TN 37831

Prices available by calling (423) $576-8401$

Available to the public from:

National Technical Information Service (NTIS)

U.S. Department of Commerce

5285 Port Royal Road

Springfield, VA 22161

(703) $487-4650$ 


\section{PREFACE}

This literature review was suggested by the Pyrolysis Activity of the IEA Bioenergy Task XIII, International Energy Agency, as a necessary means to collect and compare the known toxic effects of the condensates produced during the fast pyrolysis of biomass materials. Because of the apparent similarities of the condensates produced during fast pyrolysis, slow destructive wood distillation, and incomplete biomass combustion to form smoke and smokey flavors, this review was expanded by the author to include the extensive literature found relating to these latter two processes.

\section{ACKNOWLEDGMENTS}

The financial support to perform this review was provided jointly by the Pyrolysis Activity (chaired by Professor A.V. Bridgwater) within IEA Bioenergy, the Biomass Power Program (managed by Dr. R.L. Bain) at the National Renewable Energy Laboratory of the U.S. Department of Energy, and the Bioenergy Research and Development Program of Natural Resources Canada (managed by Mr. E.N. Hogan). This support is gratefully acknowledged. The encouragement of Drs. D. Dayton, T.A. Milne, and R. Overend of NREL in this project is also gratefully acknowledged. 


\section{Contents}

Preface $\ldots \ldots \ldots \ldots \ldots \ldots \ldots \ldots \ldots$

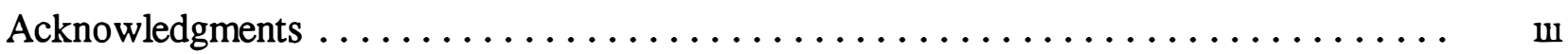

Abstract $\ldots \ldots \ldots \ldots \ldots \ldots \ldots \ldots \ldots \ldots \ldots \ldots \ldots \ldots \ldots \ldots \ldots \ldots \ldots \ldots$

Introduction $\ldots \ldots \ldots \ldots \ldots \ldots \ldots \ldots \ldots \ldots \ldots \ldots \ldots \ldots \ldots \ldots \ldots \ldots$

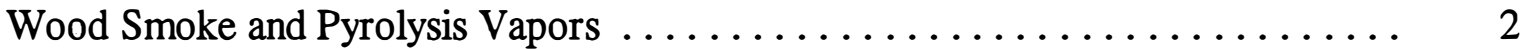

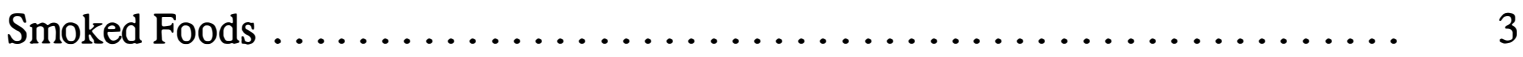

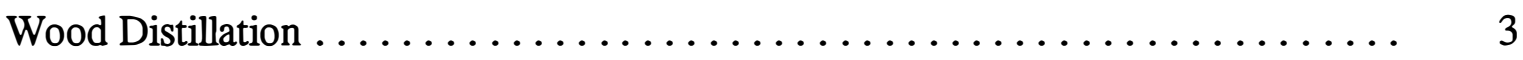

Fast Pyrolysis for Organic Liquids $\ldots \ldots \ldots \ldots \ldots \ldots \ldots \ldots \ldots \ldots \ldots \ldots \ldots \ldots \ldots \ldots$

Composition of Wood Smoke, Wood Distillates, and Fast Pyrolysis Oils . . . . . . . 5

Acute and Chronic Toxicity of Compounds Found in Fast Pyrolysis Oils . . . . . . 12

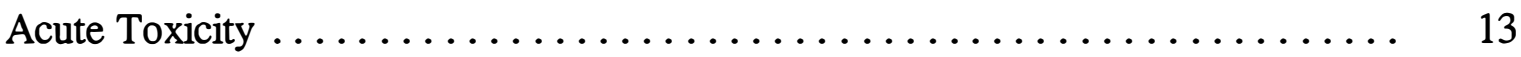

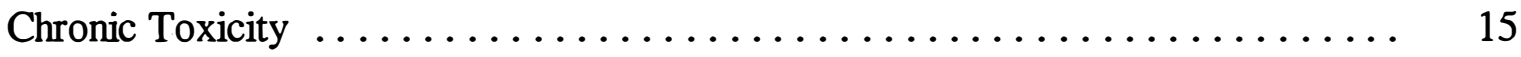

Acute Toxicity of Wood Distillates, Smoke, Liquid Smoke, and Fast Pyrolysis Oils . . . . 16

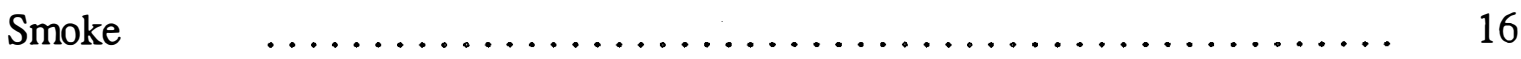

Liquid Smoke $\ldots \ldots \ldots \ldots \ldots \ldots \ldots \ldots \ldots \ldots \ldots \ldots \ldots \ldots \ldots \ldots \ldots$

Fast Pyrolysis Oils $\ldots \ldots \ldots \ldots \ldots \ldots \ldots \ldots \ldots \ldots \ldots \ldots \ldots \ldots \ldots \ldots$

Chronic Toxicity of Coal Smoke, Wood Smoke, Smoke Flavors, Wood Distillates,

Fast Pyrolysis Oils $\ldots \ldots \ldots \ldots \ldots \ldots \ldots \ldots \ldots \ldots \ldots \ldots \ldots \ldots \ldots \ldots \ldots$

Chronic Toxicity of Coal and Wood Smoke ................ 18

Chronic Toxicity of Food and Smoked Foods . . . . . . . . . . . . . . . 19

Chronic Toxicity of Biomass Fast Pyrolysis Oils and Gasifier Tars . . . . . . . 21

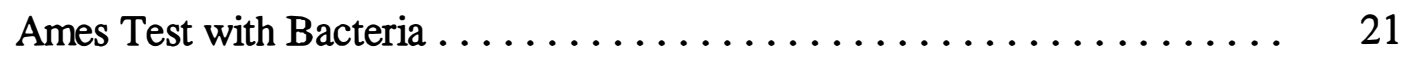

Mammalian Studies with Fast Pyrolysis Oils .............. 22

Effect of Time and Temperature on Toxicity of Pyrolysis Condensates $\ldots \ldots \ldots 23$

Material Safety Data Sheets $(\mathrm{MSDS}) \ldots \ldots \ldots \ldots \ldots \ldots \ldots \ldots \ldots \ldots \ldots \ldots$

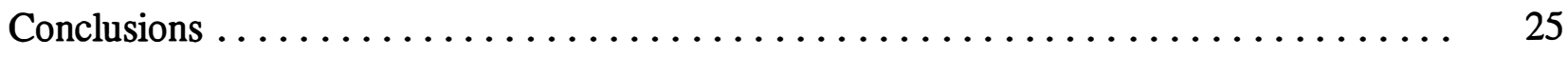

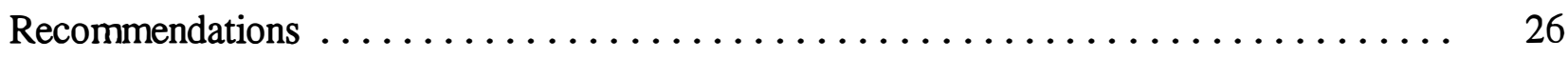

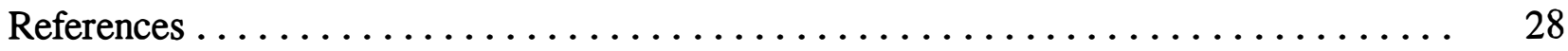




\section{Tables}

1a. Compounds Identified in Pyrolysis Products and Their Toxicity-

Page

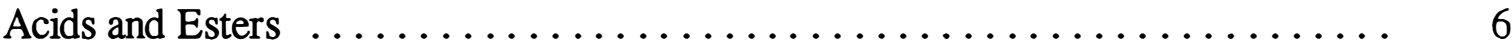

1b. Compounds Identified in Pyrolysis Products and Their Toxicity-

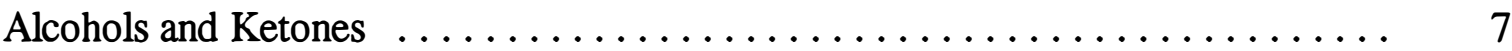

1c. Compounds Identified in Pyrolysis Products and Their Toxicity-

Aldehydes and Phenols $\ldots \ldots \ldots \ldots \ldots \ldots \ldots \ldots \ldots \ldots \ldots \ldots \ldots$

1d. Compounds Identified in Pyrolysis Products and Their Toxicity-

Guaiacols, Syringols, and Sugars ....................... 9

1e. Compounds Identified in Pyrolysis Products and Their Toxicity-

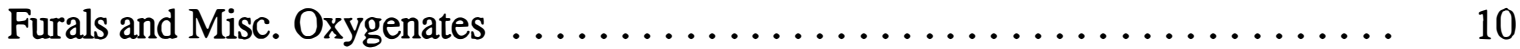

1f. Compounds Identified in Pyrolysis Products and Their Toxicity-

Alkenes, Aromatics, Nitrogen Compounds, and Mixed Oxygenates . . . . . . . 11 


\title{
A REVIEW OF THE TOXICITY OF BIOMASS PYROLYSIS LIQUIDS FORMED AT LOW TEMPERATURES
}

\author{
by \\ James P. Diebold \\ Biomass Fast Pyrolysis Consultant \\ Thermalchemie, Inc. \\ $57 \mathrm{~N}$. Yank Way \\ Lakewood, CO 80228, USA \\ dieboljc@rmii.com (303) 989-7878
}

\begin{abstract}
The scale-up of biomass fast pyrolysis systems to large pilot and commercial scales will expose an increasingly large number of personnel to potential health hazards, especially during the evaluation of the commercial use of the pyrolysis condensates. Although the concept of fast pyrolysis to optimize liquid products is relatively new, low temperature pyrolysis processes have been used over the aeons to produce charcoal and liquid by-products, e.g., smoky food flavors, food preservatives, and aerosols containing narcotics, e.g., nicotine, etc. A number of studies exist in the historical literature concerning hazards of acute and long term exposure to smoke and to the historical pyrolysis liquids formed at low temperatures.
\end{abstract}

In this review, the methods of production of smoke, smoke flavors, wood distillates, and fast pyrolysis vapors are briefly addressed. Then the reported composition of the products from these processes is compared to give the reader a feeling for their relative similarity. From the list of compounds reported to be in fast pyrolysis condensates at over $0.1 \%$ and for a few other selected compounds of interest, the toxicity of each compound is listed for which information is available.

The oral toxicity of a worst case scenario, hypothetical fast pyrolysis oil containing the maximum reported level of each compound reported is calculated and compared to toxicity data reported for pyrolysis products. It is concluded that compounds from the aldehyde and furan families found in some fast pyrolysis oils constitute the worst oral and inhalation health hazards. The chronic health effects would also be related to the aldehydes as the potential source of problems, unless the severity of the pyrolysis is so high as to create significant quantities of benzene and polycyclic aromatic hydrocarbons.

The reported toxicity of smoke, smoke food flavors, and fast pyrolysis oils is reviewed. The data found for these complex mixtures suggest that the toxicity may be less than that of the individual components. It is speculated that there may be chemical reactions that take place that serve to reduce the toxicity during aging.

The U.S. Occupational Safety and Health requirements (OSHA 1996a) for a worker's right-toknow about chemical compounds in the workplace are reviewed and suggestions are made for the generation of additional information for a Material Safety Data Sheet for fast pyrolysis oils. 


\section{Introduction}

\section{Wood Smoke and Pyrolysis Vapors}

Oxygenated organic vapors are formed during the heating of biomass with or without the presence of air at local temperatures between about $200^{\circ} \mathrm{C}$ (for several hours) and $500^{\circ} \mathrm{C}$ (in less than a second). In an ideal combustion situation, these organic vapors continue to decompose and oxidize to form carbon dioxide and water. In a less than ideal combustion, part of the organic vapors do not complete the decomposition steps and are not completely oxidized. Any organic vapors that escape destruction in the hot environment can cool and condense to form liquids or liquid aerosols known as smoke. Upon further cooling, these liquids may solidify, depending upon their composition.

In the extreme case of a lack of oxygen, i.e., pure pyrolysis, the organic vapors are not oxidized at all. However, the first formed or primary oxygenated vapors are not thermally stable and they undergo a series of reactions to form more stable compounds. The primary vapors consist of a very complex mixture of oxygenated compounds that represent the partial decomposition products of the macropolymers found in biomass, i.e., cellulose, hemicellulose, lignin, and extractives. Careful study of the progression of the decomposition of these primary pyrolysis products using Molecular Beam/Mass Spectroscopy show that in the absence of air, they tend to lose their complexity as they crack to form permanent gases and more thermally stable, organic compounds. After exposure to a more severe environment of a higher temperature, e.g., $700^{\circ} \mathrm{C}$ for 0.75 seconds, the primary vapors lose much of their complexity to become secondary vapors with a significantly reduced yield and having a more phenolic nature. At higher severities, e.g., $950^{\circ} \mathrm{C}$ for 0.75 seconds, the primary vapors are converted to tertiary vapors with only a few per cent yield and a decidedly aromatic nature containing benzene and polycyclic aromatic hydrocarbons (PAH). (Evans and Milne 1987) The condensation of these pyrolysis vapors forms liquids called fast pyrolysis oils (if essentially primary in nature) or pyrolysis tars (if essentially tertiary in nature). Persistent aerosols are also formed during condensation, which are also called smoke.

These changes in chemical nature are a gradual shift and often a sample of smoke will contain a mixture of primary, secondary, and tertiary tars, depending upon the temperature and time history of each molecule in the smoke. This shift in the composition of pyrolysis vapors has also been demonstrated by analysis of pyrolysis condensates from a variety of different pyrolysis systems operating at different temperatures with the progression of organic vapor composition being given as: mixed oxygenates at $400^{\circ} \mathrm{C}$; phenolic ethers at $500^{\circ} \mathrm{C}$; alkyl phenolics at $600^{\circ} \mathrm{C}$; heterocyclic ethers at $700^{\circ} \mathrm{C}$; and $\mathrm{PAH}$ above $800^{\circ} \mathrm{C}$ (Elliott 1986,1988 ).

Given the fact that the primary pyrolysis products are fragments of the compounds in the feedstock, the pyrolysis products will vary depending upon the feedstock used. For example, the smoke from tobacco contains nitrogen based compounds that are not found in wood smoke, i.e, nicotine, etc. This, coupled with the effect of time and temperature on the compounds to be found in smoke, results in a very wide possible chemical composition for smoke and pyrolysis 
condensates. This wide variety in composition makes it difficult to draw general conclusions to categorize these complex products as to their toxicity.

\section{Smoked Foods}

Early humans learned that when they dried their meat, that this would preserve it. One can imagine that on a rainy day, an impatient person tried to dry their meat above a smokey fire and discovered that the resulting smoked meat tasted better than sun-dried meat and later realized that it was also better preserved. Biomass smoke contains a wide variety of acids and phenolic materials, which have bactericidal, fungicidal, and anti-oxidant effects to better preserve the meat than simple desiccation (Maga 1988)(Tóth and Potthast 1984). After a little experimentation, one can imagine that it was quickly learned that smoke from some fuels tasted better than smoke from others.

As with many other processes that were historically developed, a science has emerged to better understand and to improve it. In a typical old-fashioned smokehouse, the smoke is generated by a smoldering pile of wet sawdust, usually from hardwoods such as hickory, oak, mesquite, etc. This technique produces copious quantities of smoke with very little investment, but it does have several serious drawbacks. Raw wood smoke tends to make a meat product in which the brown color is proportional to the intensity of the smoke flavor. Due to the crude nature of the smoke generation device, it is not readily controllable, which can result in a variability of flavor intensity that is not popular with most consumers. (Maga 1987) A simple ablative pyrolysis reactor using the frictional heat generated by wood pressed against a spinning metal disk was also used to produce wood smoke for food processing, which produced a smoke superior to that produced by smoldering sawdust (Anon. 1956)(Husaini and Cooper 1957).

In order to better control the smoking process, a product obtained by the ethyl acetate extraction of the aqueous phase of wood distillation condensates was marketed in the 1940's in France (Stamm and Harris 1953). Unfortunately, the smoke extract obtained from wood distillation did not contain the chemistry that turned the meat brown. Today's consumer in the USA likes a browned color, but with a lightly smoked flavor (Underwood 1992). Chemical research has shown that the browning ability is linked to the carbonyl content of the smoke flavoring (Underwood 1992), especially glyoxal (ethandial), hydroxyacetaldehyde, and methyl hydroxyacetaldehyde, which react with amino acids to form brown colors (Riha and Wendorff 1993). It has been long been known by workers in the field that fast pyrolysis oils, which contain these aldehydes, that accidentally come in contact with human skin will rapidly turn it dark brown. Fast pyrolysis oils can be used to make smoke-flavored, browning liquids for meat products (Underwood and Graham 1989). It has been shown that the desirable wood smoke flavors are to be found in the steam distillable compounds (Porter et al 1965)(Husaini and Cooper 1957). Liquid smoke preparations have been commercially in use that are made from the aqueous soluble portion of smoke, wood distillates, and fast pyrolysis oils.

\section{Wood Distillation}

In primitive charcoal processes, wood piles are covered with dirt to limit the combustion or placed in a room with limited air ventilation. Part of the pile is ignited. After some time has passed, the flow of air is stopped and the pile allowed to slowly "cook" for several days. In these 
primitive systems, no attempt is made to recover the condensates. Organic volatiles and tar vapors are formed, which condense into smoke and are allowed to escape to the environment. This gives rise to considerable local pollution. When using resinous softwoods, a devolatilized rosin melts and runs to the bottom of the kiln where it is recovered.

Historical wood distillation techniques improved on the primitive processes by collecting the organic volatiles and tars for sale as by-products. The yields of charcoal increased from better heating practices to yields of 30 to $40 \mathrm{wt} \%$ charcoal, but with only about 20 to $25 \mathrm{wt} \%$ organic condensates. Water is formed by dehydration reactions, especially in char forming reactions. This water is usually co-condensed with the organic vapors, along with the moisture present in the feedstock. The relatively large amount of water in the organic condensates resulted in a large aqueous or pyroligneous-acid phase containing dissolved tars and a small settled tar phase. During distillation of the pyroligneous-acid phase to recover the volatile methanol, acetic acid, and formic acid, a "dissolved-tar" phase would form in the bottom of the still pot. The settled-tar phase was also distilled to recover the volatiles and a pitch. If the biomass was softwood, this tacky pitch was used for adhesives and waterproofing purposes. Pitch from hardwood was greasy in nature and had little value except as a fuel. Acetic acid was recovered as calcium acetate. The calcium acetate was either heated to produce acetone and calcium carbonate or acidified to release the acetic acid. If the latter process was done in the presence of ethanol, ethyl acetate was produced. The tars were usually burned for boiler fuel.(Hawley 1923)(Klar 1925)(Stamm and Harris 1953)

Although over-heating of the oxygenated organic vapors now appears to have been responsible for the toluene and PAH reported in the early 1900's (Klar 1925), research into the heating cycle to maximize charcoal yields apparently led to the use of process conditions that had the side benefit of not co-producing aromatic hydrocarbons. The preferred heating technique for batch production of charcoal was to heat the new wood charge vigorously until the first aqueous insoluble tar was distilled over (about $275^{\circ} \mathrm{C}$ ). At that point, the wood charge was hot enough to be into the exothermic period of the carbonization process and the external heating was stopped. Most of the wood distillation occurred at temperatures below $350^{\circ} \mathrm{C}$. After the condensate formation ceased, the charcoal was externally heated to reach the desired degree of carbonization, primarily by gas forming reactions (Palmer 1915)(Hawley 1923).

In the 1910's, continuous carbonization processes were developed: a) the Seaman process involving a ram fed, inclined rotary kiln; and b) the Stafford process involving a barrel-valve fed, updraft, slow moving bed with bottom char discharge.(Stamm and Harris 1953) In the latter process, the feed was dried and preheated to $150^{\circ} \mathrm{C}$ and fed into the updraft reactor. The exothermic heat released was sufficient to maintain the center of the bed at $515^{\circ} \mathrm{C}$ with no combustion air added during steady state operation. (Nelson 1930) Note that the majority of the pyrolysis occurred well before the higher temperature was attained and that the vapors passed upward into the cooler portion of the reactor. Thus, these vapors were not exposed to the higher temperatures that are associated with the formation of secondary or tertiary tars. The absence of aromatic hydrocarbons and nitrogen compounds in the product yields was a significant feature that distinguished wood distillation from coke oven operation (Hawley 1923). (However, they were probably not able to detect ppm, nor ppb, levels of PAH.) As discussed previously, the 
vapor residence time was apparently long enough that the browning ability of the aqueous fraction was lost due to the destruction of hydroxyacetaldehyde and similar compounds.

It is encouraging that during the over 100 years or so that charcoal production in retorts and wood distillation was widely practiced, that there was not observed an increase in health problems among the distillers. (Brocksiepe 1986) However, most of that period was before there was the health awareness and concern that characterizes the modern chemical and fuel industry. So, this lack of reported problems is not proof that they did not exist. In fact, there have been studies which report the presence of mutagenic and carcinogenic activity in tars derived from charcoal operations.(Mezerette and Girard 1991).

\section{Fast Pyrolysis for Organic Liquids}

In the last 20 years, more advanced thermal processing techniques for the pyrolysis of wood and other biomass have resulted in the optimization for the production of organic vapors which can be condensed to form oxygenated, liquid pyrolysis products. These thermal processing techniques typically utilize rapid heating rates, high heat transfer coefficients, a relatively low temperature heat source, and a short residence time for the vaporized organic products. This has been demonstrated in pyrolysis reactors using various technologies, e.g., slow heating under vacuum (Roy et al 1988), fluidized bed reactors (Scott et al 1984)(Cuevas et al 1996), entrained coiled tube reactor (Brown 1996)(Brown and Black 1992), entrained tubular reactor (Novac and O'Neil 1989), entrained tubular reactors with thermofor (Graham et al 1994)(Boukis 1996), entrained ablative vortex reactors (Diebold and Scahill 1996), and ablative mill reactors (Reed 1988)(Peacocke and Bridgwater 1996). In small scale laboratory experimental units, the yields of organic liquids from wood have been reported to be as high as $70 \mathrm{wt} \%$ of the dry feedstock on a moisture free oil and wood basis (Scott and Piskorz 1984). In scaled up pyrolysis units, the organic liquid yields have been somewhat lower at between 45 and 60 wt\% (dry bases) due primarily to the cracking of organic vapors and an increase in the production of gases.

\section{Composition of Wood Smoke, Wood Distillates, and Fast Pyrolysis Oils}

In Table 1a through 1f, are listed over 74 compounds that have been reported to be in fast pyrolysis oils at concentrations of at least $0.1 \mathrm{wt} \%$, with the wt\% of the compound in the wet oil shown in column 4 (Milne, et al 1996). In addition to these compounds, certain compounds have been added to Table 1a through 1f, where it was felt that the compounds deserved consideration due to their toxicity or peculiar absence in the fast pyrolysis oils. In the second column are listed abbreviations for those authors who have reported the indicated chemical species in wood distillates: f (Fraps 1901); g (chemicals commercially available in Goos and Reiter 1946); $h$ (Hawley 1923); k (with references from the 1800's in a review by Klar 1925); s (a review by Stamm and Harris 1953); and v (a modern GC/MS analysis by Vergnet and Villeneuve 1988). In the third column are listed abbreviations for those authors who have reported the indicated chemical species in wood smoke: G (a commercial aqueous liquid smoke flavor analyzed by GC/MS in Guillén et al 1995); and gi (a lab produced, aqueous phase smoke flavor analyzed by GC/MS in Guillén and Ibargoitia 1996); $\mathrm{m}$ (wood smoke in general in reviews by Maga 1987 and 
Table 1a. Compounds Identified in Pyrolysis Products and their

Toxicity-Acids and Esters

\begin{tabular}{|c|c|c|c|c|c|c|c|c|c|c|c|c|}
\hline Compound & $\begin{array}{c}\text { Wood } \\
\text { Distillate } \\
1\end{array}$ & $\begin{array}{l}\text { Smoke } \\
\text { Flavors }\end{array}$ & $\begin{array}{c}\text { Fast Pyr. } \\
\text { Oils } \\
w t \%\end{array}$ & $\begin{array}{c}\text { Inhal. } \\
\mathrm{LC}_{50} \\
\mathrm{mg} / \mathrm{m} 3 / \mathrm{h}\end{array}$ & $\begin{array}{c}\text { Oral } \\
\mathrm{LD}_{s 0} \\
\mathrm{mg} / \mathrm{kg}\end{array}$ & $\begin{array}{c}\text { Skin } \\
\mathrm{LD}_{50} \\
\mathrm{mg} / \mathrm{kg}\end{array}$ & \begin{tabular}{|c|}
$\mathrm{PEL}$ \\
$\mathrm{TWA} 8 \mathrm{~h}$ \\
$\mathrm{mg} / \mathrm{m} 3$
\end{tabular} & $\begin{array}{l}\text { STEL } \\
\mathrm{mg} / \mathrm{m} 3 \\
\end{array}$ & $\begin{array}{r}\text { Irrit } \\
\text { skin }\end{array}$ & $\begin{array}{l}\operatorname{tant} \\
\text { eye }\end{array}$ & $\mid \begin{array}{l}\text { Mammal } \\
\mathrm{kg}, \text { Oral } \\
\mathrm{LD}_{50} 15 \mathrm{ml} \\
\text { Fst Pyr.Oil }\end{array}$ & Comments \\
\hline \multicolumn{13}{|l|}{ Acids } \\
\hline Formic (methanoic) & $\mathrm{f}, \mathrm{g}, \mathrm{h}, \mathrm{k}, \mathrm{s}, \mathrm{v}$ & gi,m,t & $0.3-9.1$ & $15000 / / .25$ & 1100 & -------- & 9 & ------- & mld & sev & 1.6 & $\mathbf{M}$ \\
\hline Acetic (ethanoic) & $f, g, h, k, s, v$ & gi,m,t & $0.5-12$ & $14000 / / 1$ & 3310 & 1060 & 25 & & sev & sev & 0.68 & $\mathrm{M}, \mathrm{R}, \mathrm{T}$ \\
\hline Propanoic & $f, g, h, k, s, v$ & gi,m & $0.1-1.8$ & --------- & 2600 & 500 & 30 & -------- & sev & sev & 0.13 & $\mathbf{M}$ \\
\hline Hydroxyacetic & $f,,, s, v$ & $\mathrm{~m}$ & $0.1-0.9$ & --------- & 1950 & ------- & ---------- & & --- & sev & 0.09 & $\cdots$ \\
\hline 2-Butenic(crotonic) & $f, g, h, k, s, v$ & $\mathrm{~m}$ & -------- & --------- & 4800 & 600 & --------- & --------- & --- & --- & ---------- & $\mathbf{M}$ \\
\hline Butanoic & $\mathrm{f}, \mathrm{g}, \mathrm{h}, \mathrm{k}, \mathrm{s}, \mathrm{v}$ & $\mathrm{t}$ & $0.1-0.5$ & --------- & 2000 & 530 & --------- & --------- & sev & sev & 0.05 & $\mathbf{M}$ \\
\hline Pentanoic (valeric) & $\mathrm{f}, \mathrm{h}, \mathrm{k}, \mathrm{s}, \mathrm{v}$ & gi,m,t & $0.1-0.8$ & $4100 / / 2$ & 600 & 700 & ------- & -------- & ---- & --- & 0.3 & --- \\
\hline 2-Me butenoic & $\mathrm{f}, \mathrm{h}, \mathrm{k}, \mathrm{s}$ & $\mathrm{m}$ & --------- & -------- & 5000 & 5000 & ------ & -------- & ---- & --- & ---- & ---- \\
\hline 4-oxypentanioc & $,, \mathrm{s}, \mathrm{v}$ & gi,m & $0.1-0.4$ & --------- & 1850 & ------ & ------- & -------- & ---- & --- & 0.04 & $-\cdots$ \\
\hline 4-hydroxypentanoic &,,,, $\mathrm{v}$ & & -------- & -------- & 8800 & 5000 & ------- & & mld & ---- & $\ldots$ & $\mathbf{M}$ \\
\hline Hexanoic (caproic) & $\mathrm{f}, \mathrm{h}, \mathrm{s}$ & $\mathrm{m}$ & $0.1-0.3$ & $4100 / / 2$ & 3000 & 630 & ------- & --- & mld & sev & 0.03 & $\mathbf{M}$ \\
\hline Benzoic & & $\mathrm{m}, \mathrm{t}$ & $0.2-0.3$ & --------- & 1700 & -------- & -------- & & mld & sev & 0.03 & $\mathbf{M}$ \\
\hline Heptanoic & & $\mathrm{m}$ & 0.3 & - & 6400 & ----- & ------- & ------- & --- & --- & 0.01 & ---- \\
\hline \multicolumn{13}{|l|}{ Esters } \\
\hline$\overline{\text { Methyl formate }}$ & $\mathrm{f}, \mathrm{h}, \mathrm{s}$ & $\mathrm{m}, \mathrm{t}$ & $0.1-0.9$ & ------- & 1622 & ----- & 250 & 375 & ---- & ---- & 0.1 & ---- \\
\hline Methyl acetate & $\mathrm{f}, \mathrm{h}, \mathrm{k}, \mathrm{s}, \mathrm{v}$ & $\mathrm{m}, \mathrm{t}$ & --------- & $97000 / / 4$ & 3705 & ------ & 610 & 760 & $\bmod$ & $\bmod$ & -------- & $\mathbf{M}$ \\
\hline Propiolactone & & & ---------- & $25 / / 6$ & 50low & ------ & ---------- & --------- & ---- & ---- & --------- & $\mathrm{M}, \mathrm{T}, \mathrm{C}_{\mathrm{o}}, \operatorname{poss}_{\mathrm{i}, \mathrm{n}}$ \\
\hline Methyl propionate & $\mathrm{f}, \mathrm{h}, \mathrm{h}, \mathrm{v}$ & $\mathrm{m}$ & ---------- & 27000//-- & 5000 & ------- & ---------- & --------- & $\bmod$ & ---- & -------- & ---- \\
\hline Butyrolactone & $\mathrm{g}, \quad, \mathrm{s}, \mathrm{v}$ & $\mathrm{m}, \mathrm{t}$ & $0.1-0.9$ & ---------- & 1540 & 5000 & ---------- & -------- & --- & ---- & 0.1 & $\mathbf{M}, \mathbf{R}, \mathrm{T}$, inad. $\mathbf{C}_{\mathbf{i}}$ \\
\hline Methyl Crotonate & & $\mathrm{m}$ & --------- & & 1600 & 10000 & --------- & --------- & $\bmod$ & $\bmod$ & d ---------- & \\
\hline Methyl n-butyrate & $\mathrm{f}, \mathrm{h}$, , & $\mathrm{m}, \mathrm{t}$ & ---------- & 18000//-- & 5000 & ------- & ---------- & --------- & $\bmod$ & $\bmod$ & I ---------- & ---- \\
\hline Valerolactone & $\mathrm{f}, \mathrm{h}, \mathrm{k}, \mathrm{s}, \mathrm{v}$ & & 0.2 & ---- & 8800 & 5000 & ---------- & ------ & mld & ---- & 0.004 & $\mathbf{M}$ \\
\hline angelicalactone & & $\mathrm{m}$ & $0.1-1.2$ & --------- & 2800 & ------ & -------- & --------- & ---- & ---- & 0.080 & ---- \\
\hline Methyl valerate & $\mathrm{f}, \mathrm{h}$, & gi,m,t & --------- & $6600 / / 2$ & ------ & ------ & 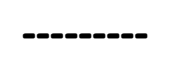 & 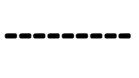 & ---- & ---- & --------. & ---- \\
\hline
\end{tabular}


Table 1b. Compounds Identified in Pyrolysis Products and their Toxicity---Alcohols and Ketones

\begin{tabular}{|c|c|c|c|c|c|c|c|c|c|c|c|}
\hline Compound & $\begin{array}{c}\text { Wood } \\
\text { Distillate } \\
1\end{array}$ & \begin{tabular}{|} 
Smoke \\
Flavors
\end{tabular} & $\begin{array}{c}\text { Fast Pyr. } \\
\text { Oils } \\
\text { wt } \%\end{array}$ & $\begin{array}{l}\text { Inhal. } \\
\mathrm{LC}_{50} \\
\mathrm{mg} / \mathrm{m} 3 / / \mathrm{h}\end{array}$ & $\begin{array}{c}\text { Oral } \\
\mathrm{LD}_{50} \\
\mathrm{mg} / \mathrm{kg}\end{array}$ & $\begin{array}{l}\text { Skin } \\
\mathrm{LD}_{50} \\
\mathrm{mg} / \mathrm{kg}\end{array}$ & $\begin{array}{c}\text { PEL } \\
\text { TWA } 8 \mathrm{~h} \\
\mathrm{mg} / \mathrm{m} 3\end{array}$ & $\begin{array}{l}\text { STEL } \\
\mathrm{mg} / \mathrm{m} 3\end{array}$ & \begin{tabular}{|} 
Irritant \\
skin leye
\end{tabular} & $\begin{array}{l}\text { Mammal } \\
\begin{array}{|l|}\mathrm{kg}, \text { oral } \\
\mathrm{LD}_{50}, 15 \mathrm{ml} \\
\text { Fst Pyr.Oil }\end{array}\end{array}$ & Comments \\
\hline \multicolumn{12}{|l|}{ Alcohols } \\
\hline Methanol & $f, g, h, k, s, v$ & $\mathrm{~m}, \mathrm{t}$ & $0.4-2.4$ & $64000 / / 4$ & 5628 & 15800 & 260 & 309 & $\bmod \bmod$ & 0.080 & M,R \\
\hline Ethanol & $, \mathrm{g},, \mathrm{,v}$ & $\mathrm{m}, \mathrm{t}$ & $0.6-1.4$ & $39000 / 10$ & 7060 & 20000 & 1900 & -------- & $\bmod s e v$ & 0.04 & $\mathrm{M}, \mathrm{R}, \mathrm{T}$ \\
\hline 2-propene-1-ol & $\mathrm{f}, \mathrm{h}, \mathrm{k}, \mathrm{s}$ & $\mathrm{m}, \mathrm{t}$ & ------- & $180 / / 8$ & 64 & 45 & $5 \mathrm{sk}$ & $10 \mathrm{sk})$ & ---- sev & ---------- & $\mathbf{M}$ \\
\hline Isobutanol & $\mathrm{f}, \mathrm{h}, \mathrm{k}, \mathrm{,v}$ & $\mathrm{m}$ & -------- & ----------- & 2460 & 3400 & 150 & -------- & $\bmod s e v$ & ---------- & $\mathrm{M}, \mathrm{T}$, \\
\hline 3-Methyl-1-butanol &,,, $\mathrm{s}$ & & -------- & ----------- & 1300 & 3212 & 360 & 450 & $\bmod \bmod$ & | ---------- & $\mathrm{T}$, \\
\hline $\begin{array}{l}\text { Ethylene glycol } \\
\text { Ketones }\end{array}$ & & $\mathrm{m}$ & $0.7-2.0$ & 10876//-- & 4700 & 9530 & 125 & ------- & mld mod & 0.080 & M,R \\
\hline Acetone & $\mathrm{f}, \mathrm{h}, \mathrm{k}, \mathrm{s}, \mathrm{v}$ & $\mathrm{m}, \mathrm{t}$ & 2.8 & $50100 / / 8$ & 5800 & 20000 & 590 & ------ & mld sev & 0.091 & M,R \\
\hline 2-Butenone & & $\mathrm{d}$ & --------- & $7 / / 4$ & 30 & -------- & --- & & ---- ---- & --------- & $\mathbf{M}$ \\
\hline 2-Butanone (MEK) & $f, g, h, k, s, v$ & $\mathrm{~m}$ & $0.3-0.9$ & $23500 / / 8$ & 2737 & 6480 & 590 & 885 & mld ---- & 0.06 & M,R \\
\hline 2,3 butandione &,,,,,,$v$ & & --------- & ---------- & 1580 & ------- & --------- & --------- & ---- ---- & ---------- & ---- \\
\hline Cyclo pentanone & $\mathrm{f}, \mathrm{h}, \mathrm{s}, \mathrm{v}$ & $\mathrm{m}, \mathrm{t}$ & --------- & ---------- & ----- & ------- & --------- & ------- & sev mod & | --------- & ---- \\
\hline 2-pentanone & $\mathrm{f}, \mathrm{,}, \mathrm{,}, \mathrm{v}$ & $\mathrm{m}$ & -------- & $7000 / 4 l o w$ & 1600 & 6500 & 700 & 875 & mld ---- & & ------ \\
\hline 3-pentanone & ,h, , , & $\mathrm{m}$ & --------- & --------- & 2140 & 20000 & 705 & -------- & mld mod & | --------- & $\mathbf{M}$ \\
\hline 2-cyclopentenone &,,,,,$v$ & gi,m & --------- & --------- & --.----- & -------- & ---------- & -------- & ---- ---- & ---------- & $\mathrm{T}$ \\
\hline 2,3 pentenedione &,,,,,$v$ & $\mathrm{~m}, \mathrm{t}$ & $0.2-0.4$ & ---------- & ----- & -------- & ---------- & --------- & ---- ---- & ---------- & ---- \\
\hline 3Me2cyclopenten2ollone & $f_{,, y, s}$ & $\mathrm{~m}$ & $0.1-0.6$ & ---------- & 1400 & ------ & --------- & s & ---- ---- & 0.08 & ---- \\
\hline Me-cyclopentanone & $\mathrm{f}, \mathrm{h}, \mathrm{s}, \mathrm{s}$ & $\mathrm{m}, \mathrm{t}$ & --------- & --------- & ------ & ------ & ---------- & --------- & ---- ---- & ---------- & ---- \\
\hline 2-hexanone & & $\mathrm{m}$ & --------- & $9789 / / 4$ & 2590 & 4800 & 20 & -------- & mld mld & ---------- & ---- \\
\hline Cyclo hexanone & & $\mathrm{m}$ & trace & $9789 / / 4$ & 1535 & 948 & 100sk & & mld sev & ---------- & $\mathbf{M}, \mathbf{R}$ \\
\hline methylcyclohexanone & $\mathrm{f}$, & & --------- & & 2140 & 1635 & 230 & 45(sk) & ---- ---- & --------- & --- \\
\hline 2-Et-cyclopentanone & & $\mathbf{t}$ & $0.2-0.3$ & ------ & -------- & ------ & --------- & --------- & ---- ---- & ---------- & ---- \\
\hline Dimethylcyclopentanone & & $\mathrm{m}$ & 0.3 & --------- & ------- & ------ & --------- & --------- & ---- ---- & ---------- & --- \\
\hline trimethylcyclopentenone & & gi & $0.1-0.5$ & --------- & -------- & -------- & --------- & --------- & ---- ---- & --------- & ---- \\
\hline trimethylcyclopentanone & & $\mathrm{m}$ & $0.2-0.4$ & --------- & ------ & ------- & --------- & & ---- ---- & & --- \\
\hline
\end{tabular}


Table 1c. Compounds Identified in Pyrolysis Products and their Toxicity---Aldehydes and Phenols

\begin{tabular}{|c|c|c|c|c|c|c|c|c|c|c|c|c|}
\hline Compound & $\begin{array}{c}\text { Wood } \\
\text { Distillate } \\
1\end{array}$ & $\begin{array}{r}\text { Smoke } \\
\text { Flavors }\end{array}$ & $\begin{array}{c}\text { Fast Pyr. } \\
\text { Oils } \\
w t \%\end{array}$ & $\begin{array}{l}\text { Inhal. } \\
\mathrm{LC}_{50} \\
\mathrm{mg} / \mathrm{m} 3 / / \mathrm{h}\end{array}$ & $\left|\begin{array}{c}\text { Oral } \\
\mathrm{LD}_{50} \\
\mathrm{mg} / \mathrm{kg}\end{array}\right|$ & $\begin{array}{c}\text { Skin } \\
\mathrm{LD}_{50} \\
\mathrm{mg} / \mathrm{kg}\end{array}$ & $\begin{array}{c}\text { PEL } \\
\text { TWA 8h } \\
\text { mg/m3 }\end{array}$ & $\begin{array}{l}\text { STEL } \\
\mathrm{mg} / \mathrm{m} 3\end{array}$ & $\begin{array}{r}\text { Irri } \\
\text { skin }\end{array}$ & $\begin{array}{l}\text { tant } \\
\text { leye }\end{array}$ & $\mid \begin{array}{l}\text { Mammal } \\
\mathrm{kg}, \text { oral } \\
\mathrm{LD}_{50}, 15 \mathrm{ml} \\
\text { Fst Pyr.Oil }\end{array}$ & Comments \\
\hline \multicolumn{13}{|l|}{ Aldehydes } \\
\hline Formaldehyde & , h, , , & $\mathrm{m}, \mathrm{t}$ & $0.1-3.3$ & 203//--- & 100 & 270 & 1.2 & 10 & sev & sev & 6.2 & $\mathrm{M}, \mathrm{R}, \mathrm{T}$, prob. $\mathrm{C}_{\mathrm{i}, \mathrm{n}}$ \\
\hline Acetaldehyde & $f, g, h, k, s, v$ & gi,m,t & $0.1-8.5$ & $24000 / / 4$ & 661 & 3540 & 180 & 270 & mld & sev & 2.4 & $\mathrm{M}, \mathrm{R}, \mathrm{T}$,poss. $\mathrm{C}_{\mathrm{i}, \mathrm{n}}$ \\
\hline 2-Propenal (acrolein) & & $\mathrm{m}$ & $0.6-0.9$ & $300 / / 0.5$ & 46 & 562 & 0.25 & 0.8 & sev & sev & 4 & $M, R$, inad. $C_{i, n}$ \\
\hline 2-Butenal &,,$v$ & $\mathrm{~m}$ & trace & $4000 / / 0.5$ & 240 & 380 & 6 & -------- & mld & ---- & 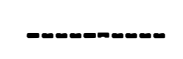 & $\mathbf{M}$, inad. $C_{i}$ \\
\hline 2-Methyl-2-butenal & & gi,m & $0.1-0.5$ & --------- & ----- & ------- & ---------- & -------- & ---- & ---- & ---------- & ---- \\
\hline pentanal & $\mathrm{f}, \mathrm{h}, \mathrm{h}, \mathrm{v}$ & $\mathrm{m}$ & 0.5 & --------- & 4581 & 4857 & 175 & -------- & $\bmod$ & sev & 0.02 & $\mathbf{M}$ \\
\hline Ethanedial & ---- & ---- & $0.9-4.6$ & --------- & 1100.0 & 6600.0 & -------- & ------ & mld & sev & 0.78 & $\mathbf{M}$ \\
\hline \multicolumn{13}{|l|}{ Phenols } \\
\hline Phenol & $\mathrm{f}, \mathrm{h}, \mathrm{s}, \mathrm{v}$ & $\mathrm{G}, \mathrm{gi}, \mathrm{m}, \mathrm{t}$ & $0.1-3.8$ & $316 / /--$ & 317 & 850 & 19(sk) & ------ & sev & sev & 2.2 & $M, R, T$, inad. $C_{i}$ \\
\hline 2-methyl phenol & $\mathrm{f}, \mathrm{h}, \mathrm{s}, \mathrm{v}$ & $\mathrm{G}, \mathrm{gi}, \mathrm{m}, \mathrm{t}$ & $0.1-0.6$ & $179 / / 2$ & 121 & 890 & $22(\mathrm{sk})$ & ----- & sev & sev & 0.9 & $\mathrm{M}, \mathrm{T}$ \\
\hline 3-methyl phenol & $\mathrm{f}, \mathrm{h}, \mathrm{s}, \mathrm{v}$ & $\mathrm{G}, \mathrm{gi}, \mathrm{m}, \mathrm{t}$ & $0.1-0.4$ & --------- & 242 & 2050 & $22(\mathrm{sk})$ & -------- & sev & sev & 0.3 & $\mathrm{M}, \mathrm{R}, \mathrm{T}$ \\
\hline 4-methyl phenol & $\mathrm{f}, \mathrm{h}, \mathrm{,}, \mathrm{v}$ & $\mathrm{G}, \mathrm{gi}, \mathrm{m}, \mathrm{t}$ & $0.1-0.5$ & ----.--- & 207 & 301 & $22(\mathrm{sk})$ & & --- & ---- & 0.5 & $\mathrm{M}, \mathrm{T}$ \\
\hline 2,3 dimethyl phenol & , , , s,v & $\mathrm{G}, \mathrm{gi}, \mathrm{m}, \mathrm{t}$ & $0.1-0.5$ & --------- & 56 i.v. & ----- & ------- & -------- & ---- & ---- & 1.7 & --- \\
\hline 2,4 dimethyl phenol & $\mathrm{f}, \mathrm{h}, \mathrm{s}, \mathrm{v}$ & $\mathrm{G}, \mathrm{gi}, \mathrm{m}, \mathrm{t}$ & $0.1-0.3$ & --------- & $3200^{\circ}$ & 1040 & -------- & & ---- & --- & 0.02 & $\mathrm{~T}$, \\
\hline 2,5 dimethyl phenol & $,,, s, v$ & $\mathrm{G}, \mathrm{gi}, \mathrm{m}, \mathrm{t}$ & $0.2-0.4$ & --------- & 444 & ------ & ------- & ------- & ---- & ---- & 0.2 & $\mathrm{~T}$ \\
\hline 2,6 dimethyl phenol & $,,,, s, v$ & G,gi,m,t & $0.1-0.4$ & --------- & 296 & 1000 & -------- & 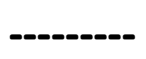 & --- & --- & 0.3 & $\mathrm{~T}$ \\
\hline 3,5 dimethyl phenol & $\mathrm{f}, \mathrm{h}, \mathrm{s}, \mathrm{v}$ & $\mathrm{m}, \mathrm{t}$ & --------- & --------- & 608 & ------- & ---- & ------- & ---- & ---- & 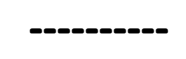 & $\mathrm{T}$ \\
\hline 2-ethyl phenol & $\mathrm{f},,$, & $\mathrm{G}, \mathrm{gi}, \mathrm{m}, \mathrm{t}$ & $0.1-1.3$ & ------- & 600 & ------- & ---------- & 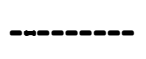 & ---- & ---- & 0.41 & $\mathrm{~T}$ \\
\hline 2,4,6 triMe phenol & ------ & $\mathrm{m}$ & 0.3 & --------- & 10000 & ------ & --------- & --------- & ---- & --- & 0.006 & --- \\
\hline 1,2 diOH benzene & $\mathrm{f}, \mathrm{h}, \mathrm{k}, \mathrm{s}, \mathrm{v}$ & $\mathrm{G}, \mathrm{gi}, \mathrm{m}, \mathrm{t}$ & $0.1-0.7$ & ---------- & 260 & 800 & 20 (sk) & --------- & --- & ---- & 0.5 & $M, R$, inad. $C_{i}$ \\
\hline 1,3 diOH benzene & --------- & $\mathrm{m}$ & $0.1-0.3$ & $>7800 / / 1$ & 301 & 3360 & 45 & 90.0 & $\bmod$ & sev & 0.2 & $\mathrm{M}, \quad \mathrm{T}$, inad. $\mathrm{Ci}$ \\
\hline 1,4 diOH benzene & --------- & ----- & $0.1-1.9$ & ---------- & 320 & 5970 & 2 & --------- & sev & ---- & 1.1 & M,R, T,inad. $C_{i}$ \\
\hline 4-methoxy catechol & --------- & $\mathrm{G}, \mathrm{gi}, \mathrm{m}, \mathrm{t}$ & 0.6 & & ------ & ------ & -------- & --------- & ---- & ---- & -----. & --- \\
\hline $1,2,3$ tri-OH-benzene & , h, , s & $\mathrm{t}$ & 0.6 & ---------- & 1600 & ------ & -------- & --------- & sev & $\bmod$ & 0.1 & $\mathbf{M}, \mathbf{R}$ \\
\hline
\end{tabular}


Table 1d. Compounds Identified in Pyrolysis Products and their Toxicity---Guaiacols, Syringols, and Sugars

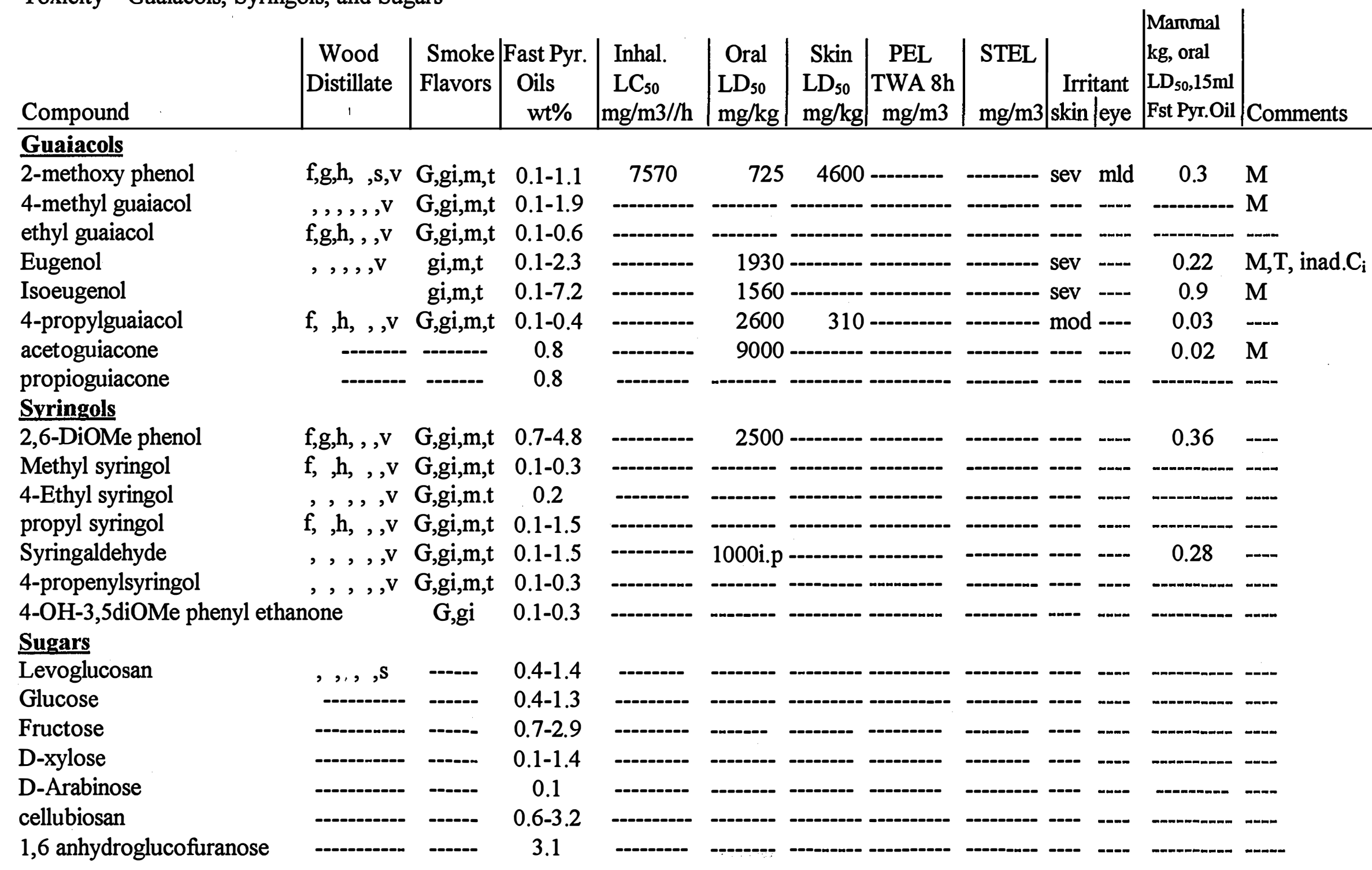


Table 1e. Compounds Identified in Pyrolysis Products and their Toxicity---Furans and Misc. Oxygenates

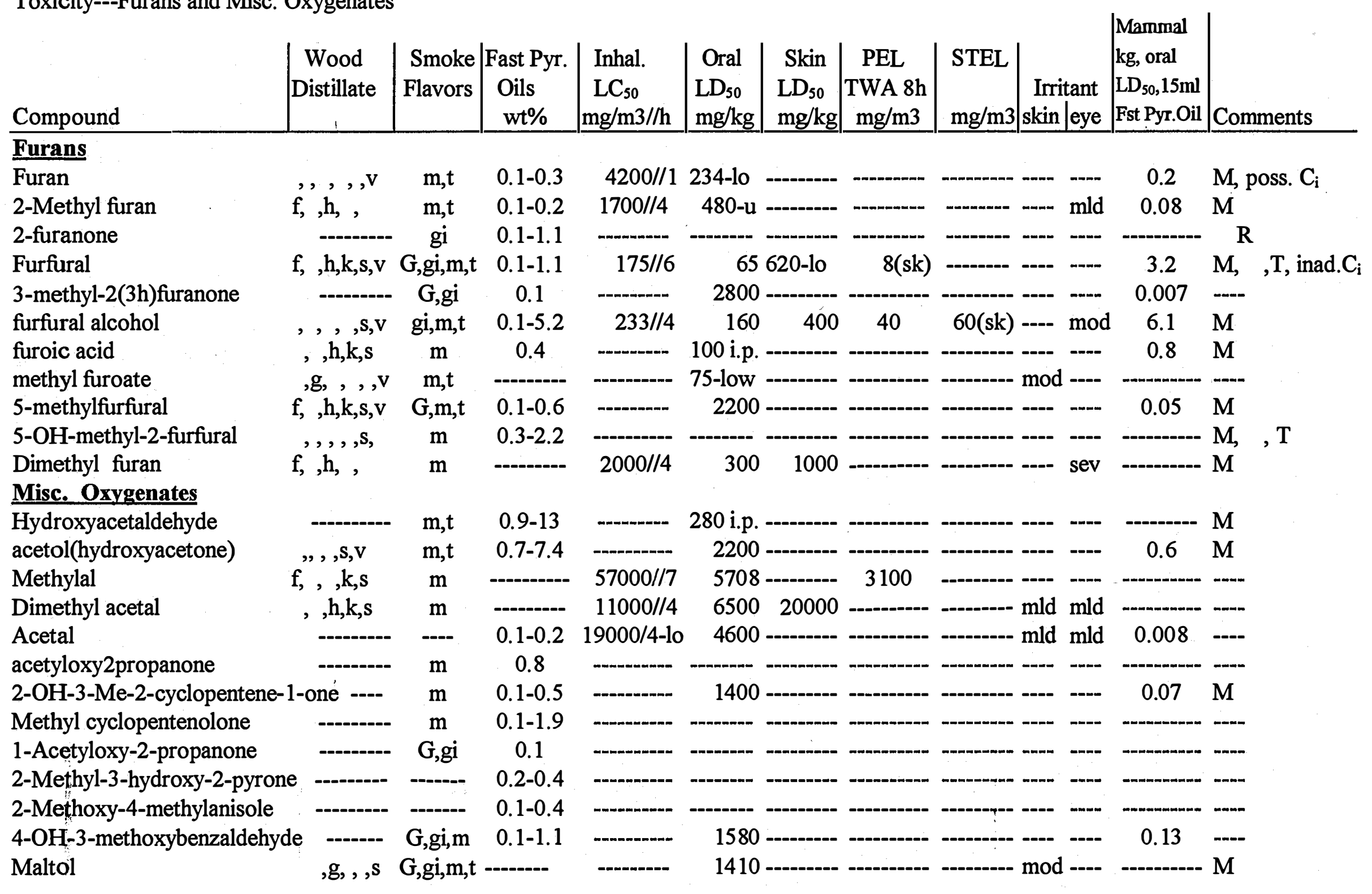


Table 1f. Compounds Identified in Pyrolysis Products and their Toxicity---Alkenes, Aromatics, Nitrogen Compounds, and Mixed Oxygenates

\begin{tabular}{|c|c|c|c|c|c|c|c|c|c|c|c|c|}
\hline Compound & $\begin{array}{c}\text { Wood } \\
\text { Distillate }\end{array}$ & $\begin{array}{l}\text { Smoke } \\
\text { Flavors }\end{array}$ & \begin{tabular}{|c} 
Fast Pyr. \\
Oils \\
wt\%
\end{tabular} & $\begin{array}{l}\text { Inhal. } \\
\mathrm{LC}_{50} \\
\mathrm{mg} / \mathrm{m} 3 / / \mathrm{h} \\
\end{array}$ & $\left|\begin{array}{c}\text { Oral } \\
\mathrm{LD}_{50} \\
\mathrm{mg} / \mathrm{kg}\end{array}\right|$ & $\begin{array}{c}\text { Skin } \\
\mathrm{LD}_{50} \\
\mathrm{mg} / \mathrm{kg}\end{array}$ & $\begin{array}{c}\text { PEL } \\
\text { TWA } 8 \mathrm{~h} \\
\mathrm{mg} / \mathrm{m} 3\end{array}$ & $\begin{array}{l}\text { STEL } \\
\mathrm{mg} / \mathrm{m} 3 \\
\end{array}$ & & $\begin{array}{l}\text { itant } \\
\text { leye }\end{array}$ & $\begin{array}{l}\text { Mammal } \\
\text { kg, oral } \\
\text { LD }_{50,15 \mathrm{ml}} \\
\text { Fst Pyr.Oil }\end{array}$ & Comments \\
\hline \multicolumn{13}{|l|}{ Alkenes } \\
\hline$\overline{\text { 2-methyl propene }}$ & -------- & --..-- & - & $620 / / 4$ & ------ & -------- & - & - & $-\cdots$ & --- & --------- & ---- \\
\hline dimethylcyclopentene & -........- & -..--..- & 0.7 & - & -..--..- & -..--- & --------- & ------.-- & $-\cdots$ & --- & -------- & ---- \\
\hline alpha-pinene &, , ,s & -..-- & - & -...- & 3700 & --------- & ---------- & --------- & sev & $-\cdots$ & -...-.-.- & --- \\
\hline dipentene &, , s & - & 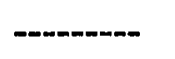 & -.---.- & ------ & ----- & --------- & --------- & $\bmod$ & ---- & $\cdots$ & --- \\
\hline \multicolumn{13}{|l|}{ Aromatics } \\
\hline Benzene & $,,, \mathrm{k}, \mathrm{,}, \mathrm{v}$ & $\mathrm{m}$ & -------- & $32000 / / 7$ & 930 & $>8263$ & 3 & 15 & $\bmod$ & sev & ---------- & $\mathrm{M}, \mathrm{R}, \mathrm{T}, \mathrm{C}_{\mathrm{i}, \mathrm{n}}$ \\
\hline Toluene & $\mathrm{f}, \mathrm{h}, \mathrm{k}, \mathrm{s}, \mathrm{v}$ & $\mathrm{G}, \mathrm{m}$ & --------- & $>26700 / 1$ & 636 & 12124 & 375 & 560 & $\bmod$ & sev & ---------- & $M, R$, inad. $C_{i}$ \\
\hline Xylenes & $\mathrm{f}, \mathrm{h}, \mathrm{k}, \mathrm{s}$ & & -------- & $5000 / / 4$ & 4300 & --------- & 100 & 150 & $\bmod$ & sev & --------- & $\mathrm{R}$, inad. $\mathrm{C}_{\mathbf{i}}$ \\
\hline Naphthalene & $,,,, s, v$ & $\mathrm{~m}, \mathrm{t}$ & --------- & ---------- & 533 & 20000 & 50 & 75 & mld & mld & ---------- & $\mathrm{M}, \mathrm{R}, \mathrm{T}$ \\
\hline Phenanthrene & & $\mathrm{t}$ & --------- & --------- & 700 & -------- & 0.2 & --------- & ---- & --- & ---------- & $\mathrm{M}, \mathrm{T}$, inad. $\mathrm{C}_{\mathrm{i}}$ \\
\hline Fluoranthene & & $\mathrm{t}$ & & ---------- & 2000 & 3180 & ---------- & --------- & ---- & ---- & ---------- & $\mathrm{M}, \mathrm{T}$, inad. $\mathrm{C}_{\mathrm{i}}$ \\
\hline Chrysene &,, $\mathrm{k}$ & $\mathrm{t}$ & --------- & --------- & ------- & ------ & 0.2 & --------- & ---- & ---- & ---------- & $M,, T$, inad. $C_{i}$ \\
\hline \multicolumn{13}{|l|}{ Nitrogen Cmpds } \\
\hline ammonia & , h, h,s & & --------- & $2447 / / 4$ & ------- & -------- & -------- & 27 & ---- & ---- & ---------- & $\mathbf{M}$ \\
\hline methyl amine & $\mathrm{f}, \mathrm{h}, \mathrm{k}, \mathrm{s}$ & $\mathrm{m}$ & --------- & $2400 / / 2$ & & & & & sev & --- & ---------- & $\mathbf{M}$ \\
\hline pyridine & $, \mathrm{h}, \mathrm{s}$ & $\mathrm{m}$ & --------- & --------- & 891 & 1121 & 15 & --------- & mld & sev & ---------- & $\mathbf{M}$ \\
\hline methyl pyridine & , h, ,s & & -------- & $1220 / / 4$ & 1290 & 270 & ----- & --------- & & sev & --- & ---- \\
\hline \multicolumn{13}{|l|}{ Mixed Oxygenates } \\
\hline Wood Creosote & , h,h,s & - & --.-- & -. & 885 & ------ & -------- & ----- & --- & - & 21.2 & $-\cdots$ \\
\hline Liquid Smoke & & Maga88 & -..-- & - & 3550 & -------- & -------- & --------- & --- & -..- & 5.3 & --- \\
\hline Fast Pyrolysis Oils & & & Gratson & $3100 / / 4$ & ----- & $>2000$ & -------- & --------- & $\mathrm{mld}$ & sev & --- & --- \\
\hline
\end{tabular}


1988); and $t$ for the detailed review by Tóth and Potthast 1984. Note that the differences in the method of generation of wood distillates and a wood smoke are not clear cut and may overlap. However, it does not appear that there are new oxygenated species that appear as a result of air in the formation of smoke, with the possible exception of pentones, hexones, and oxidized phenolics. Column three of Table 1 represents a selected short list of ompounds found in wood smoke, as well over 400 compounds have been identified in literature reviews (Maga 1987 and 1988). A detailed discussion of the chemistry of the compounds found in wood smoke is found in Tóth and Potthast (1984).

An inspection of Table 1 reveals that there are some compounds reported by nearly all of the authors cited. However, some compounds that were found by only one of the referenced authors and this may have been due to differences in the feedstock, thermal processes used, or the analytical processes used. As noted in the fourth column, many of these compounds are found in fast pyrolysis oils in widely varying, but quite small amounts. Based on the information in the first four columns of Table 1, it appears that there are a great many similarities in the chemical composition of wood distillates, wood smoke and liquid smoke, and fast pyrolysis oils. Certainly all of them contain organic acids, aldehydes, phenolics, esters, ketones, and furans. It is worth noting that in a fast pyrolysis oil produced at $550^{\circ} \mathrm{C}$, the level of individual aromatic compounds, e.g., benzene, toluene, xylene, naphthalene, etc., was reported to be less than $0.01 \mathrm{wt} \%$, with the total amount of reported aromatic hydrocarbon compounds of $0.06 \mathrm{wt} \%$ (Williams and Horne 1995). In pyrolysis oils made under vacuum conditions at $200^{\circ} \mathrm{C}$ to $448^{\circ} \mathrm{C}$, the $\mathrm{PAH}$ were also very low. It would appear safe to assume that any toxicity problems observed with wood distillates or wood smoke should be looked for in fast pyrolysis oils. It is also obvious that the chemical composition of fast pyrolysis oils is very different than coal tar materials (Kirk and Othmer 1983) or wood gasifier tars made at high temperatures (Pakdel and Roy 1991), which are mostly polycyclic aromatic hydrocarbons (PAH).

\section{Acute and Chronic Toxicity of Compounds Found in Fast Pyrolysis Oils}

The majority of Table 1 deals with the reported toxicity of pure chemical compounds, as reported in the Registry of Toxic Effects of Chemical Substance (RTECS 1993), which is maintained by the National Institute for Occupational Safety and Health (NIOSH), a part of U.S. Department of Health and Human Services. This registry is available on microfiche from the U.S. Government Printing Office for a nominal charge, as well as on CD-ROM from commercial sources for a substantial investment. This registry contains the toxicity of over 118,000 different chemical compounds along with the references to the source of that toxicity information. In addition, a large number of these compounds are cross-referenced to various synonyms for the same compound, making it easier to find organic compounds that have many different names. It was attempted to purge Table 1 of multiple listings of the same compound under different names and to use the most descriptive name where possible. Toxicity information is also available for chemical compounds of a commercial nature in Material Safety Data Sheets (for examples see MSDS 1986) that are made available to the purchasers of chemicals in the U.S. However, many of the chemicals reported to be in fast pyrolysis oils are not of a commercial nature and do not have MSDS listings in the data bases that were accessed. For the purposes of consistency, it was 
decided to use the NIOSH registry (RTECS 1993) for this review, as it appeared to be the most comprehensive.

\section{Acute Toxicity}

In Table 1 are listed the lethal dosages to mammals for a $50 \%$ mortality rate $\left(\mathrm{LC}_{50}\right)$ of each compound by inhalation in $\mathrm{mg} / \mathrm{m}^{3}\left(1 \mathrm{mg} / \mathrm{m}^{3} \sim 1 \mathrm{ppm} \mathrm{wt}\right)$ for a specified time of exposure, by oral ingestion $\left(\mathrm{LD}_{50}\right)$ and by dermal exposure $\left(\mathrm{LD}_{50}\right)$ in $\mathrm{mg} / \mathrm{kg}$ animal weight. In these columns, the abbreviations "low" indicates the data is for the lowest lethal dose rather than for $50 \%$ lethality, i.v. is intravenous dosage, i.p. is for intraperitoneal dosage, and " $u$ " is unknown route of dosage. Usually the oral lethality data selected was for rats and the dermal lethality data for rabbits, but lethality data for mice and other small animals was used rather than leave the space blank. The literature is far from being complete concerning the toxicity of chemical compounds. These lethality values are given by NIOSH (and are presented here) only as a general guide to the toxicity of the chemical compounds. Because it is known that lethal doses vary between species and individuals, extrapolation of these numbers to humans is definitely not recommended to be relied upon. In addition, it is known that some persons have, or can develop after repeated exposures, a sensitivity to certain compounds that trigger allergic and other adverse reactions.

The Permissible Exposure Level (PEL) is established by the U.S. Occupational Safety and Health Administration (OSHA 1993) as the maximum concentration in air allowable for an eight hour exposure period for all persons. The Short Term Exposure Limit (STEL) is the maximum allowable exposure concentration for a short time of 15 minutes during the eight hour exposure period. The abbreviation "sk" indicates that if skin contact is possible, that this must be taken into account when calculating the PEL and STEL.

In Table 1, the lowest PEL is for acrolein (2-propenal) at $0.25 \mathrm{mg} / \mathrm{m}^{3}$, followed by formaldehyde at $1 \mathrm{mg} / \mathrm{m}^{3}$. The maximum reported acrolein content reported in fast pyrolysis oils was $0.9 \mathrm{wt} \%$ and that of formaldehyde was $3.3 \mathrm{wt} \%$, so this would limit the concentration of whole pyrolysis oils to $28 \mathrm{mg} / \mathrm{m}^{3}$ based on acrolein and to $30 \mathrm{mg} / \mathrm{m}^{3}$ based on formaldehyde content. Acrolein can form by the reaction of formaldehyde with acetaldehyde. Acrolein has a high reactivity due to conjugation of the carbonyl group with a vinyl group. It reacts easily with alcohol groups to form acrolein acetals and alkoxypropionaldehydes. It slowly reacts with itself to form the dimer and with water to form 3-hydroxypropionaldehyde. Acrolein is very irritating to the eyes and is very highly toxic to fish and to the bacteria present in water-treatment facilities. It has a very low odor threshold of $0.23 \mathrm{mg} / \mathrm{m}^{3}$ and is said to be its own waming agent. (Kirk and Othmer 1991)

In addition, the smokey odor thresholds of the phenolics are quite low, e.g., with guaiacol at $0.025 \mathrm{mg} / \mathrm{m}^{3}$, 4-methyl guaiacol at $0.09 \mathrm{mg} / \mathrm{m}^{3}$, and syringol at $1.85 \mathrm{mg} / \mathrm{m}^{3}$ (Maga 1986, 1988). Using just the smokey odor of guaiacol, at the lowest reported concentration of $0.1 \mathrm{wt} \%$ in fast pyrolysis oils, a concentration of $25 \mathrm{mg}$ fast pyrolysis $0 \mathrm{il} / \mathrm{m}^{3}$ can be detected with the human nose. Because guaiacol is only one of many odoriferous compounds present, it would appear that the operators will be alerted to the presence of dangerous concentrations of pyrolysis oil vapors.

Thus, the pyrolysis oils and pyrolysis gases are odorized with smoke odors, and are identifiable from just the guaiacol content at fast-pyrolysis oil concentrations below those required by OSHA 
relative to the PEL for acrolein. This is analogous to the t-butyl mercaptan used commercially to odorize natural gas for safety purposes. During the operation of pyrolysis systems, a major lethal threat is from the odorized pyrolysis gases containing odorless carbon monoxide leaking into the workspace. It is important that the pyrolysis operators, exposed to the immediate threat of exposure to pyrolysis gases, not wear organic face masks which would remove the warning odorants but not the carbon monoxide from the air they breathe (unless air-supplied face masks are used).. In work spaces where carbon monoxide in the pyrolysis gases is not a threat, the operators will be well advised to wear organic vapor masks if exposed to pyrolysis oil vapors. However, OSHA has a recommended air sampling procedure that analyzes for both formaldehyde and acrolein, that would ensure the safety of the operators in case their olfactory senses have become overloaded, thus eliminating the recognition of the waming signs of the odors. (OSHA 1996c) There are also commercially available hand-held carbon-monoxide detectors that are very useful to detect dangerous levels of carbon monoxide, with or without the smoke odors.

In order to gain an appreciation for the lethal dose numbers, the oral $\mathrm{LD}_{50}$ listed for ethanol of $7060 \mathrm{mg} / \mathrm{kg}$ corresponds to $0.746 \mathrm{~L}$ of $95 \%$ grain alcohol for an average man weighing $75 \mathrm{~kg}$ (165 lbs.), which has been tragically demonstrated among inexperienced young drinkers as being close to correct. In the second to the last column is listed the size of mammal, that would have a $50 \%$ mortality rate by orally ingesting $15 \mathrm{ml}$ (one U.S. tablespoon) of a pyrolysis oil containing the maximum amount of each chemical reported to have been found in pyrolysis oils in column four. By this measure, larger animals will be killed with the more toxic chemicals that are present in higher concentrations.

It is seen that the most dangerous chemical known to be present in pyrolysis oils is formaldehyde, with the potential to kill a $6.2-\mathrm{kg}$ mammal $50 \%$ of the time with an oral dose of a tablespoon of pyrolysis oil. The next most dangerous chemicals are: furfural alcohol with the potential to kill a $6.1-\mathrm{kg}$ mammal $50 \%$ of the time; acrolein and a $4-\mathrm{kg}$ mammal; furfural and a 3.2-kg mammal; acetaldehyde and a 2.4-kg mammal; phenol and a 2.0-kg mammal; 2,3 dimethyl phenol and a $1.7-$ $\mathrm{kg}$ mammal; formic acid and a $1.6-\mathrm{kg}$ mammal; and 1,4 dihydroxy phenol and a 1.1-kg mammal. If the reported toxicity of the chemicals reported in Table 1 were to be additive, the tablespoon of the hypothetically worst fast pyrolysis oil (containing $147 \mathrm{wt} \%$ of the toxic chemicals at their maximum reported values) would be able to kill a $39-\mathrm{kg}$ mammal $50 \%$ of the time, or normalized to $100 \mathrm{wt} \%$ would imply a $27-\mathrm{kg}$ mammal (or an estimated oral $\mathrm{LD}_{50}$ of $\sim 700 \mathrm{mg} / \mathrm{kg}$ ). Obviously, the toxic effects could be synergistic and therefore more deadly, but due to different metabolistic pathways for the different chemicals, it is conjectured that fast pyrolysis oil will prove not to be this toxic to mammals. It appears that small amounts of pyrolysis oils that might be accidentally ingested through eating with dirty hands, etc., is not a serious concern (Gratson 1994).

There are several compounds listed in Table 1, which were not reported in Milne, et al, to be present at over $0.1 \mathrm{wt} \%$, but were included in the table because of their toxicity, or reported presence in wood distillates and wood smoke, or because they are an extension of a homologous series of compounds that are present in pyrolysis oils. An example of this is $\beta$-propiolactone with a molecular weight of 72 and an $\mathrm{LC}_{50}$ of only $25 \mathrm{mg} / \mathrm{m}^{3}$ with an exposure time of 6 hours. $\mathrm{MB} / \mathrm{MS}$ spectra for the pyrolysis of wood at $500^{\circ} \mathrm{C}$ does show a small peak at $\mathrm{M} / \mathrm{Z} 72$ (Evans 
and Milne 1987), but this could be due to the presence of organic compounds other than $\beta$ propiolactone, e.g., propenic acid, or pentanes. $\beta$-propiolactone was not reported to be found in smoke or pyrolysis products, but was included in the table due to its toxicity and to the reported presence of other lactones. The literature (Sax 1979) indicates it decomposes rapidly at its boiling point of $155^{\circ} \mathrm{C}$, so it may not survive the pyrolysis process if it were to be formed. $\beta$ propiolactone also is known to have a short half life in water and to polymerize upon standing at room temperature (NTP 1996b), so it could also transesterify with the other organic esters, acids, and alcohols present in the pyrolysis oils. These considerations would indicate that $\beta$ propiolactone probably is not in fast pyrolysis oils.

\section{Chronic Toxicity}

In Table 1 in the right-most column, titled "Comments," are abbreviations for long term health effects that have been reported in the Registry: $M$ for mutagenic, $R$ for reproductive, $T$ for tumorigenic, and $C$ for cancerous to humans with various subscripts to indicate the source of information: "i" for the International Agency for Research of Cancer (IARC 1996), " $n$ " for the U.S. National Toxicology Program (NTP 1996), "o" for the U.S. Occupational Safety and Health Administration (OSHA 1996b). Prefixes to the letter "C" are "prob" for probably cancerous to humans, "poss" for possibly or potentially cancerous to humans, and "inad" for inadequate data available to classify as cancerous to humans. The details of these tests are beyond the scope of this review, but if one were to be contemplating the recovery or concentration of these compounds that have been noted to have long term health effects, it would prudent to carefully review the references listed in the Registry (RTECS 1993) to determine the proper safety precautions to take.

Only a very few compounds are listed on the "Known to be Cancerous" lists, but of the compounds listed in Table 1, only benzene appears on the IARC and NTP lists. (Surprisingly, also on the IARC list of things known to be carcinogenic to humans is wood dust, which is a potential feedstock to make fast pyrolysis oil. The IARC monograph on wood dust was published quite recently (IARC 1995) and apparently OSHA still officially views wood dust as inert.) OSHA's list of 13 carcinogens (OSHA 1996b) includes $\beta$-propiolactone (in concentrations above $1 \mathrm{wt} \%$ or vol\%), but $\beta$-propiolactone has not been reported to be in fast pyrolysis oils, wood distillates, nor in wood smoke.

However, OSHA does have special rules for polycyclic aromatic hydrocarbons found in coalcoking oven emissions, benzene, and formaldehyde. These special rules include very low allowable inhalation exposure levels and the posting of signs and labels indicating that a cancer hazard exists with benzene and coal-coke oven emissions and that a potential cancer hazard exists with formaldehyde. The allowable concentration of coal tar pitch volatiles, anthracene, benzo(a)pyrene (BaP), phenanthrene, acridine, chrysene, and pyrene are quite low at $0.2 \mathrm{mg} / \mathrm{m}^{3}$ (OSHA 1993) Fortunately, these aromatic compounds have been found to be present in only trace amounts, or not at all, in fast pyrolysis oil made at $550^{\circ} \mathrm{C}$, e.g., $6.5 \mathrm{ppm}$ anthracene, $<0.0$ ppm BaP, $11.4 \mathrm{ppm}$ phenanthrene, $54 \mathrm{ppm}$ alkyl phenanthrenes, $<0.0 \mathrm{ppm}$ chrysene, and $0.6 \mathrm{ppm}$ pyrene (Williams and Horne 1995). However, the potential is there to make them from wood or fast pyrolysis vapors if they are inadvertently exposed to high temperatures for even a brief period(Evans and Milne 1987)(Elliott 1986 and 1988). 
Compounds in Table 1 and of interest to producers of fast pyrolysis oils that are listed as probably or possibly carcinogenic to humans by the International Agency for Research of Cancer (IARC 1996) or reasonably anticipated to be carcinogenic by the National Toxicology Program (NTP 1996a) include: acetaldehyde (NTP, IARC), benzofuran (IARC), creosotes (IARC), formaldehyde gas (NTP, IARC), furan (IARC), and propiolactone (NTP, IARC). It will also be of tangential interest to producers of fast pyrolysis oils that marine diesel fuels, residual fuel oils, gasoline, diesel engine exhausts, gasoline engine exhausts, 13 to 15 four- and five-ring polycyclic aromatics including $\mathrm{BaP}$, carbon black, and respirable ceramic fibers and glass wool fibers are also listed by NTP or IARC in these categories of hazards.

Compounds in Table 1 that have been unclassifiable with respect to cancer due to inadequate data for humans and inadequate or limited data for animals are acrolein, anthracene, fluorene, phenanthrene, chrysene, $\gamma$-butyrolactone, 1,2 dihydroxy benzene, 1,3 dihydroxy benzene, chrysene, crotonaldehyde, eugenol, furfural, d-limonene, phenol, toluene, and xylene. Also of interest to the producer of fast pyrolysis oils is the inclusion in this list of crude oil, light diesel fuels, light fuel oils, and jet fuels. Only one compound, caprolactum, is listed as "probably not carcinogenic to humans. (IARC 1996)

The lack of an entry in Table 1 does not imply that the material is safe, but rather that there was no information available concerning the toxicity of that compound. It interesting to note that phenol, cresols, aldehydes, organic acids and alcohols appear to be mutagenic, including acetic acid which is found in table vinegar at the $5 \mathrm{vol} \%$ level (similar to the concentration found in fast pyrolysis oils). It is quite likely that the effects of concentrated compounds are different than those of diluted compounds, e.g., the effects of glacial acetic acid versus vinegar or concentrated $95 \%$ ethanol compared to beer at 3 to $6 \%$ ethanol.

\section{Acute Toxicity of Wood Distillates, Smoke, Liquid Smoke, and Fast Pyrolysis Oils}

At the bottom of Table $1 \mathrm{f}$ is listed the reported toxicity of coal creosote (Kirk and Othmer 1983), wood creosote (RTECS 1993), liquid smoke (Maga 1988), and fast pyrolysis oils (Gratson 1994). Wood creosote is a heavy distillate obtained from the water insoluble (settled) tars recovered during wood distillation (Klar 1925). Creosote is used as a wood preservative and today is derived primarily from coal, which results in an increased level of toxic properties. The toxicity of creosote is from its mixed phenolic content. It is interesting to note in passing that "PAH are primarily skin and eye irritants but are tolerated internally... and (if ingested) pass through largely unchanged..."(Kirk and Othmer 1983)

\section{Smoke}

Studies of the smoke inhalation hazards faced by forest firefighters led to the conclusion that the acute toxic effects were caused by acrolein (propenal), benzene, carbon monoxide, formaldehyde, and respiratory particulates present in the smoke. A technique for monitoring the accumulated exposure to wood smoke involved the use of cost-effective dosimeters that measured exposure to carbon monoxide. (Reinhardt et al 1994) 


\section{Liquid Smoke}

In the case of liquid smoke, Polish commercial liquid smoke preparations of unspecified concentration were studied with $\mathrm{LD}_{50}$ for rats reported to be between 3550 and $4010 \mathrm{mg} / \mathrm{kg}$ (Fitko el al 1977a). In studies with pigs fed daily doses of liquid smoke between 200 and 1400 $\mathrm{mg} / \mathrm{kg}$, a decrease in weight gain (in spite of increased food consumed) was observed with the higher dosages (Fitko et al 1977b). Similar results were observed with rats fed with between 0.1 and $2 \%$ liquid smoke in their food. The mammalian species sensitivity to oral ingestion of liquid smoke varied by a factor of over 25 , with an $\mathrm{LD}_{50}$ of $192 \mathrm{mg} / \mathrm{kg}$ for cats, $2750 \mathrm{mg} / \mathrm{kg}$ for mice, $3750 \mathrm{mg} / \mathrm{kg}$ for rats, and over $5000 \mathrm{mg} / \mathrm{kg}$ for pigs. (Kozlowski 1969)

\section{Fast Pyrolysis Oils}

The two fast pyrolysis oils tested for toxicity that were reported by Gratson (1994) were made with the vortex reactor at NREL from oak or pine. These were considered to be primary oils, although the temperatures in the vapor cracker were higher at 460 to $510^{\circ} \mathrm{C}$, than has been used since that time to maximize oil yields. The inhalation tests were conducted in smoke chambers in which the oil was atomized and the animals then breathed the aerosols. This concentration is much higher than would be voluntarily tolerated by an operator and corneal eye damage was noted. For example, to achieve the $\mathrm{LD}_{50}$ aerosol suspension of $3100 \mathrm{mg} / \mathrm{m}^{3}$, would require that $75 \mathrm{ml}$ of pyrolysis oil be atomized in a room $3 \mathrm{~m}$ by $4 \mathrm{~m}$ by $2.5 \mathrm{~m}$. It has been documented that primitive cooking with an unvented wood fire indoors resulted in only $22 \mathrm{mg} / \mathrm{m}^{3}$ of smoke, an atmosphere which was tolerated by the cook but considered to be very polluted by the medical observer (Mumford et al 1987).

The dermal toxicity tests did not show any acute toxic effects within 15 days from application of fast pyrolysis oils at a dosage of $2000 \mathrm{mg} / \mathrm{kg}$ to the skin of each of ten rabbits for 24 hours prior to washing with water, which covered approximately $15 \%$ of the body surface area. After the 15 days had passed, the animals were sacrificed and autopsied. No visible abnormalities were observed during the study period, nor during the autopsies. (Gratson 1994)

Tests in which $0.1 \mathrm{ml}$ of undiluted pyrolysis oil was applied in the eye of rabbits, showed the pyrolysis oil to be very aggressive and resulted in corneal damage to eight out of nine animals 21 days after dosing. Washing the eye with water immediately the application of the pyrolysis oil reduced and delayed the maximum adverse effects. (Gratson 1994) The aggressiveness to the eye is not surprising, given the high content of organic acids, aldehydes, and phenols, which were shown in Table 1 to have a similar effect. Adequate eye protection from splashes of pyrolysis oil is very important.

Based on these acute exposures, the toxicity testing consultant recommended that the MSDS contain a waming label which stated that fast pyrolysis oils are a hazardous substance to the eye and to inhalation exposure, but are not hazardous to dermal exposure. He concluded that these oils could be handled safely on a routine basis, using personnel protective gear, such as rubber gloves, goggles, and protective clothing. (John H. Butala in Gratson 1994) 


\section{Chronic Toxicity of coal smoke, wood smoke, smoke flavors, wood distillates, fast pyrolysis oils}

Chronic toxicity effects imply long term damage to the body. Examples of this type of toxicity are the increased incidence over an extended period of time of liver damage experienced by some heavy drinkers and of lung cancer experienced by some tobacco smokers. The former damage appears to be related to the continual overburdening of the metabolic mechanisms of removal of toxic compounds, whereas the latter appears to be the accumulation of carcinogenic compounds and exposure to cancer promoting chemicals over the years. Due to the complex nature of human existence and the multiple sources of toxic compounds, it is difficult to definitively state the cause of many human illnesses and bodily malfunctions. In the case of carcinogens, however, four- and five-ring polycyclic aromatics have been recognized as being responsible for the initiation of some cancers, with $\mathrm{BaP}$ being used as a marker for the presence of over $17 \mathrm{PAH}$ compounds. The growth of the cancerous cells is enhanced by chemicals called promoters, e.g., volatile phenols, aldehydes, and acids found in tobacco smoke.(Wynder and Hoffmann 1968)

Although tests with mammals, e.g. mice, rats, and rabbits, are preferred for chronic testing, it is relatively expensive. It is much cheaper and faster to expose bacteria to suspected carcinogens and then look for mutated colonies, which is known as the Ames test (Ames et al 1975). Because some intermediate products of the metabolism of toxic chemicals are more biologically active than the original chemical, an mammalian liver enzyme (S9) is usually added to a fraction of the samples to simulate what would happen during metabolism by a mammal. (Elliott 1986)

\section{Chronic Toxicity of Coal and Wood Smoke}

The chronic effects of exposure to coal smoke deposits or soot were correlated with an increase in scrotal cancer among chimney sweeps in London in 1775. These chronic effects also were observed in workers making coal briquettes using pitch dust and those exposed to crude coal tar. By $1930, \mathrm{BaP}$ recovered from coal-tar pitch was recognized as a strong carcinogen. Coal-tar pitch is recovered from the bottoms of the distillation of crude coal tar during the production of benzene, coal oil, and creosote. This pitch is a solid at ambient temperatures and the carcinogenicity among workers has been primarily from long-term, continual exposure to the finely divided solid pitch dust. Coal liquids which contained $\mathrm{BaP}$ were found to cause cancers when applied to mice. However, neither occupational exposure to the coal-derived creosote treating of wood, nor to vapors from the use of coal pitch and tars in road paving and roofing resulted in recognized increases in cancer rates. (Kirk and Othmer 1983)

The relative chronic toxicity of coal and wood smoke to human populations has been documented in different rural Chinese communes, in which "smoky" coal, "smokeless" coal, or wood was used as the fuel for cooking and heating. The smoky coal was a medium-volatile bituminous coal with $23.5 \mathrm{wt} \%$ ash. The smokeless coal contained $40 \%$ less volatiles than the smoky coal, but had a high ash content of $49 \%$. The stoves used were very primitive and consisted of shallow unvented pits in the floor of the homes. This resulted in high indoor smoke pollution (PM10) levels of 10 to $24 \mathrm{mg} / \mathrm{m}^{3}$ when burning smoky coal, 1 to $2 \mathrm{mg} / \mathrm{m}^{3}$ when burning smokeless coal, and $22 \mathrm{mg} / \mathrm{m}^{3}$ when burning wood. Thus, the amount of smoke was comparable when buming either smoky coal or wood. The smoke from the smokeless coal was primanily inorganic ash, compared to the 
organic nature of smoke from wood (phenolics, aliphatic aldehydes, alcohols, and relatively few $\mathrm{PAH}$ ) and smoky coal (PAH and methylated PAH). (Mumford et al 1987)

The Chinese communes buming smoky coal had lung cancer mortality rates in 1973-1975 as high as 152 per 100,000 , compared to 2.3 per 100,000 for the communes burning only wood (67\%) and smokeless coal (33\%). (In 1970, the lung cancer mortality rate in the U.S. was many times higher than the wood buming communes, with 54 for males and 12 for females per 100,000 in the U.S.) Air samples taken from the homes during cooking cycles showed that the smoke from smoky coal had much smaller diameter particles which enhanced their retention in the lung after inhalation. In addition, the levels of BAP and other PAH were much higher in the coal smoke than in the wood smoke. In dermal tests with mice, with a smoky-coal-smoke extract as an initiator and using a tumor promoter (12-0-tetradecanoylphorbol-13-acetate (TPA)) for 26 weeks produced three and a half times more papillomas (benign skin tumors or warts) than a similar extract from wood smoke used in the same manner. Using no tumor promoters, an extract of the smoky coal smoke applied twice weekly produced skin carcinomas in $35 \%$ of the animals after 52 weeks, whereas the animals in the control group and those exposed to the extract from wood smoke had no carcinomas. It was concluded that domestic open-fire wood smoke had little association with human lung cancer, whereas the data suggested that coal smoke was a cause of lung cancer. (Mumford et al 1987)

In a related study using cancer-prone mice, only $17 \%$ of the mice in the control group developed lung cancer after 15 to 19 months, whereas $46 \%$ of the mice exposed to wood smoke in a polluted indoor environment developed lung cancer, compared to $90 \%$ of the mice exposed to coal smoke. With rats, the incidences of lung cancer in the control group were much lower with $0.9 \%$, compared with rats exposed to wood smoke with $0 \%$ and exposed to coal smoke with $67 \%$. Although the carcinogenicity of coal smoke was clearly established, the effect of wood smoke appeared to be species dependent.(Liang et al 1988)

However, chronic exposure to wood smoke has been linked to a decrease in lung function in children and to increased chronic lung diseases in Nepal (Pierson et al 1989).

\section{Chronic Toxicity of Food and Smoked Foods}

The PAH content of food has attracted attention in recent years, with analytical techniques developed that are capable of measuring down to 0.1 parts per billion $\left(10^{\circ}\right)$. At this sensitive level of analysis, there is a considerable background of PAH to be found in our environment.

For example, the level of $\mathrm{PAH}$ in lettuce grown outside was found to be $94 \mathrm{ppb}$, whereas greenhouse grown head lettuce had only 8 ppb (Wickstrom et al 1986). Likewise, grain dried with flue gases can absorb $\mathrm{PAH}$ and be sources of carcinogens. The relative amount of $\mathrm{BaP}$ in flue-dried grain was reported to be a function of the fuel used, with lignite $>$ lignite coke $>$ fuel oil $>$ natural gas (Fritz 1974). The combustion mode in effect during the exposure of food (hot dogs) to cooking was also important with a wood fire depositing an average of $41 \mathrm{ppb} \mathrm{PAH}$, compared to wood embers of $4 \mathrm{ppb} \mathrm{PAH}$, charcoal of $0.2 \mathrm{ppb}$ PAH, electric oven with no PAH detected, and pan frying with no PAH detected. (Larsson et al 1983) 
During the charcoal broiling of beef steaks, $\mathrm{PAH}$ can be formed when the rendered fat drops onto the hot coals and is pyrolyzed with the resulting PAH vapors condensing on the cooling meat. The hotter the charcoal, the more PAH is formed. Levels of $8 \mathrm{ppb} \mathrm{BaP}$ in a total of $87 \mathrm{ppb} \mathrm{PAH}$ were detected in the "well-done" cooked meat, with the lack of nitrogen-containing polycyclic organic compounds showing that the protein in the meat was not pyrolyzing in this example. In a $0.34 \mathrm{~kg}(12 \mathrm{oz}$.) steak cooked in this manner, the amount of $\mathrm{BaP}$ present was said to be equivalent to that in the smoke of 185 cigarettes (Lijinsky and Shubik 1964), although others later placed a much higher level of $\mathrm{BaP}$ in cigarette smoke so as to only require about 1 cigarette for that amount of $\mathrm{BaP}$ (Shephard 1982). Barbequing is viewed by the U.S. Department of Agriculture and the Barbeque Industry Association as not a major source of carcinogens (assuming some moderation in consumption and not charring the meat), even though there is a higher than normal incidence of lung cancer among restaurant chefs (Benson 1992).

The flavor of wood smoke is added to many foods through the use of commercial liquid products derived from wood smoke, wood distillation, and fast pyrolysis. In the 1960's, it occurred to the producers of these flavors that the levels of PAH should be kept at a very low level in order to reduce the chronic hazards of its use, i.e., cancer. During the manufacture of these flavors, the pyrolysis condensates have sufficient water in them such that an aqueous phase and an organic tar phase separate. The flavors are derived from the aqueous phase. (Underwood 1992, Underwood and Graham 1989, Pszczola 1995) A sample of "Charsol" (made by Red Arrow) was analyzed and found to have no detectable $\mathrm{BaP}$ nor other known carcinogens, although a total of $6 \mathrm{ppb}$ $\mathrm{PAH}$ were present. This result led to the suggestion that the use of liquid smoke flavors was safer than the traditional smoke house methods (Lijinsky and Shubik 1965), which appears to be the current philosophy of two of the major producers of liquid smoke in the U.S.: Red Arrow (Underwood 1992) and Hickory Specialties (Pszczola 1995).

The efficiency of the partitioning of PAH between the aqueous phase and the organic tar phase was studied and found to be quite good. Seven aqueous smoke flavors and four tar samples were obtained from four anonymous manufacturers. Although $\mathrm{BaP}$ was not found in the aqueous flavors, one of them did contain the carcinogen 4-methyl benzo(a)pyrene at $2 \mathrm{ppb}$. In contrast, the four tar samples had BaP concentrations of $25,340,3700$, and $3800 \mathrm{ppb}$. It was concluded that the processing of smoke into aqueous solutions did produce a safer product. (White, et al 1971)

In a recent study of smoked cheeses, 12 cheeses were selected for their being processed with smoke-vapor or liquid-smoke techniques. They were purchased in retail stores in Wisconsin. In the smoke vapor technique, the aerosols are filtered out or electrostatically removed, allowing only the vapors to reach the food being smoked. No BaP was detected in any of the 12 samples, with a lower detection limit of $0.1 \mathrm{ppb}$. (Riha, et al 1992) This implies that aerosol-free vapors from fast pyrolysis oils at smoke house temperatures $\left(15\right.$ to $80^{\circ} \mathrm{C}$ (Maga 1988)) will also not contain $\mathrm{BaP}$, even if $\mathrm{BaP}$ is present in the condensed oils or smoke. Since $\mathrm{BaP}$ has an estimated boiling point of over $450^{\circ} \mathrm{C}$ and therefore a low vapor pressure at these temperatures, this is not a surprising conclusion, especially given the presence of the liquid aerosol acting as an absorbent for the $\mathrm{BaP}$ to reduce the vapor pressure even further by dilution. 
The mutagenicity of smoke condensates, used for food flavors and preservatives made from several hardwoods from Nigeria in western Africa, was studied and found to not correlate with the PAH content of the condensates. The seven quantified PAH included pyrene, BaP, benzo(a)anthracene, Benz(k)fluoranthene, benzo(b)chrysene, benzo(g,h,I)perylene, and dibenzo(a,e)pyrene. Mixtures of these PAH in concentrations typical of the smoke condensates did not induce mutations. (Asita et al 1991) This does not necessarily absolve the PAH, since it has been reported that the addition of $\mathrm{BaP}$ to a tobacco tar did not increase its tumorigenic activity either, whereas a mixture of $17 \mathrm{PAH}$ present in tobacco tars did so. (Wynder and Huffinann1968) Only a few of the PAH are implicated as mutagens or carcinogens (IARC 1996).

The stomach tumor promoting ability of a commercial hickory smoke condensate was investigated with rats that had tumors initiated with N-methyl-N'-nitro-N-nitrosoguanidine. The tumor growth rate was enhanced with rat diets containing $5 \%$ of the condensate, but not with 1.25 nor $2.5 \%$. This suggests a weak tumor promoting effect with the liquid smoke (Shichino et al 1992) and the benefits of moderation. It was independently concluded that the amount of phenolics in food treated with liquid smoke was below a toxicological relevant level (Sternitzke et al 1992).

\section{Chronic Toxicity of Biomass Fast Pyrolysis Oils and Gasifier Tars}

Ames Test with Bacteria As reported in detail in Elliott (1986) and summarized in Elliott (1988), the chronic toxicity of biomass fast pyrolysis oils was tested, as a function of the severity of the pyrolysis or gasification process. The primary pyrolysis oils tested included a softwoodderived primary pyrolysis oil fraction recovered from the second condenser (packed scrubber) from the NREL vortex pyrolysis system during Run 41 . The primary pyrolysis oil vapors in NREL Run 41 entered the vapor cracker at $485^{\circ} \mathrm{C}$ and cooled to $400^{\circ} \mathrm{C}$ as they passed through the 7-m transfer line prior to entering the char cyclone. Also tested was a secondary wood tar that had been produced by passing primary pyrolysis vapors at $530^{\circ} \mathrm{C}$ through a well insulated char cyclone and then into the heated transfer tube held at $750^{\circ} \mathrm{C}$ with a residence time of about 0.3 seconds. (Diebold 1984) The primary oil from NREL had an oxygen content of $40 \mathrm{wt} \%$ on a dry basis, whereas the secondary tar had only $4 \mathrm{wt} \%$ oxygen. Elliott also tested a reconstituted whole primary oil having an oxygen content of $41 \mathrm{wt} \%$ made from hardwood using the various condensates collected with the Georgia Tech entrained flow reactor system (Kovac and O'Neil 1989) operated with a temperature range of $477^{\circ} \mathrm{C}$ and $425^{\circ} \mathrm{C}$, as well as tar and aqueous condensates from various gasifiers operating in the range of 800 to $900^{\circ} \mathrm{C}$.

In the mutagenicity tests reported by Elliott, various strains of bacteria, salmonella typhimurim (TA 98, TA 100, TA 1595 and TA 1537), were tested with and without the liver enzyme S9. No mutagenic activity was observed for the primary pyrolysis oils, the NREL secondary tar, nor with aqueous phase organics (even those formed at high temperatures). The tars formed at $800^{\circ} \mathrm{C}$ to $900^{\circ} \mathrm{C}$ showed a significant increase in the revertants over those found in the background samples. However, at the higher concentrations of the NREL secondary tar samples applied to the bacteria, more of them died due the bactericidal nature of these materials. The dead bacteria were incorrectly counted as revertants by the automated counting instrumentation, necessitating the hand counting of the revertants for the NREL secondary tar sample. (Elliott 1986 and 1988) 
Fast pyrolysis oils made from spruce or poplar from the Waterloo fluidized-bed fast pyrolysis unit at $500^{\circ} \mathrm{C}$ and a $0.6-$ to 0.7 -second vapor residence time were tested with salmonella typhimurim strains TA 98 and TA 100 for signs of mutagenicity with and without S9 activation. The oils were quite dilute, as tested. The two oils were toxic, but not mutagenic to TA 98, with or without the S9 activation. Both oils were mutagenic to TA 100 strain, with and without S9 activation. (Scott 1987)

In a later series of Ames tests at SITEK Laboratories, Rockville, MD, a softwood (Southern Pine) derived pyrolysis oil made in the NREL vortex reactor (Run 150) with the vapor cracker at $510^{\circ} \mathrm{C}$ and a hardwood (oak) derived pyrolysis oil (NREL Run 154) made with the vapor cracker at $460^{\circ} \mathrm{C}$ were also tested. Both oils were considered toxic to the cultures at levels of $550 \mu \mathrm{g} / \mathrm{plate}$ without promotion and $1000 \mu \mathrm{g}$ softwood oil/plate and $5000 \mu \mathrm{g}$ hardwood oil/plate with promotion with TPA. Both of the oils were considered to have tested positively for mutagenic activity at concentrations below their toxic levels with or without promotion. However, the number of revertants per $\mu \mathrm{g}$ with the pyrolysis oils were only about $0.02 \%$ to $0.2 \%$ of those seen with BaP. (Gratson 1994) Note that this mutagenic result for primary oils is different than the negative result observed by Elliott (1986 and 1988), even though the same strains of bacteria and similar procedures were used.

The primary NREL pyrolysis oils used in the study reported by Gratson (1994) would appear to have had processing severities a little higher than the primary NREL oil used by Elliott, but clearly much less severe than the secondary NREL oil used by Elliott (1986 and 1988). It is not obvious why the primary oil and the cracked secondary NREL oil used by Elliott (1986 and 1988) should have had no mutagenic effects, whereas the primary oils used in the study reported by Scott and by Gratson would have mutagenic effects.

Mammalian Studies with Fast Pyrolysis Oils The effect of pyrolysis liquids on the skin of mice to initiate tumors was evaluated by Elliott (1986 and 1988) with a subset of the liquids above. Pyrolysis oils were diluted with acetone (1g oil per ml acetone) and painted on the skin of each mouse at dosage levels of $25 \mathrm{mg} /$ mouse. After two weeks had passed, the mice received twice weekly applications for 6 months of $5 \mu \mathrm{g}$ of the promoter 12-0-tetradecanoyl-phorbol-13acetate (TPA) diluted in $50 \mu \mathrm{L}$ acetone. It was found that the mice treated with the NREL primary fast pyrolysis oil fraction had a low tumor incidence that was judged not to be statistically different than the negative control group that had only acetone painted on them. In contrast to the Ames tests, the mice initiated with NREL secondary tars formed at $750^{\circ} \mathrm{C}$ did have a significantly higher number of tumors. The mice having the most tumors had been initiated with tertiary tars formed at 800 to $900^{\circ} \mathrm{C}$, which contained more BaP than the $25 \mu \mathrm{g}$ of BaP painted on the mice in the positive control group. The number of tumors observed correlated with the $\mathrm{BaP}$ content of the high temperature tertiary tars of around $3800 \mathrm{ppm}(0.38 \mathrm{wt} \%) \mathrm{BaP}$.

The NREL primary oil used in Elliott's tumor initiating study which appeared to have no tumor initiating power had no reported $\mathrm{BaP}$ in it, but the significant figures of the data suggests that $\mathrm{BaP}$ could have been present at levels lower than $50 \mathrm{ppm}(0.005 \mathrm{wt} \%)$. Recent analyses by Williams and Horne (1995) of a fast pyrolysis oil produced at $550^{\circ} \mathrm{C}$ in a fluidized bed, showed $0.0 \mathrm{ppm}$ $\mathrm{BaP}$ present, interpreted as meaning at least below $0.05 \mathrm{ppm} \mathrm{BaP}$.) Analyses of vacuum pyrolysis 
oils formed with a maximum hearth temperature of $448^{\circ} \mathrm{C}$, showed a BaP content of $0.2 \mathrm{ppm}$ in the oil from one of six condensers (Pakdel and Roy (1995). It appears that the analysis techniques, used by the food industry to achieve a sensitivity down to $0.1 \mathrm{ppb}$, have not been employed in the analyses of fast pyrolysis oils.

The two oils from the Waterloo pyrolysis unit used in the Ames Mutagenicity testing were also tested with ovary cell cultures from Chinese hamsters for chromosome damage. Over very narrow ranges of dilution, both pyrolysis oils exhibited chromosome damage to the ovary cells, both with and without the metabolic activation. (Scott 1987)

\section{Effect of Time and Temperature on Toxicity of Pyrolysis Condensates}

It is well known to researchers who produce fast pyrolysis oils, that the oils are initially very pungent and very irritating to the eyes. With time, the vapors above pyrolysis oils become more mellow and are not as irritating to the eyes. Although some of this observed qualitative change may have been due to just a physical loss of the offending volatile vapors, it has been documented that toxic unsaturated compounds in smoke condensates generated at $450^{\circ} \mathrm{C}$, e.g., the very toxic methyl vinyl ketone (2-butenone), "disappeared" with time, even while stored in tightly stoppered bottles at $25^{\circ} \mathrm{C}$. This "disappearance" appears to be a reaction phenomena to form other compounds, probably higher molecular weight materials by chemical double-bond condensation or other mechanisms. It has been shown in wood smoke condensate that within $72 \mathrm{~h}$ after production, the 2-butenone present at $2 \mathrm{~h}$ had virtually disappeared and methyl formate and methyl acetate had formed. The formation of methyl formate occurred over a period of only 70 hours, but the methyl acetate formation was still ongoing after 700 hours. Similar rates of formation of these esters were observed with simple mixtures of methanol with formic and acetic acids. (Doerr et al 1966)

Fresh gaseous volatiles formed during high temperature drying processes of wood at $170^{\circ} \mathrm{C}$ and allowed to condense directly onto the cultures were found to be mutagenic, but the same material after conventional adsorption-extraction procedures was not mutagenic. (Kurttio et al 1990)

It is very possible that early in the life of a pyrolysis oil, the relatively toxic aldehydes initially present react with alcohols to form the less toxic hemi-acetals and acetals. The aldehydes can also react with themselves to form resins. Formaldehyde is well known to react with phenolics to attach the methylol group or to form a methylene bridge between two phenolics. Based on the disappearance of 2-butenone in condensed smoke, it is probable that similar reactions occur with other toxic unsaturated hydrocarbons, e.g. acrolein, 2-butenal, crotonaldehyde, 2-propene-1-ol, etc. The oral $\mathrm{LD}_{50}$ of toxic acrolein is listed in Table 1 as $46 \mathrm{mg} / \mathrm{kg}$, whereas the acrolein dimer is much safer with an oral $\mathrm{LD}_{50}$ of $4920 \mathrm{mg} / \mathrm{kg}$ (RTECS 1993). Although not reported to be present in fast pyrolysis oils, $\beta$-propiolactone is said to homopolymerize upon standing at room temperature (NTP 1996b).

Thus, the fast pyrolysis oil immediately after formation may contain more toxic unsaturated oxygenates, aldehydes, and other compounds, than after the condensates have been allowed time 
to react toward their thermodynamic equilibrium concentrations. Aging at elevated temperatures would allow this equilibrium to be achieved at faster rates, but would have the disadvantage of also increasing the viscosity at a faster rate. This aging phenomena is speculated to result in the freshly formed fast pyrolysis oils being more of a health hazard to the pyrolysis operators, than to the end users after the oil has aged at least several days during storage and shipping. These speculations need to be investigated by GC/MS to understand the chemistry and then by mutagen activity testing.

\section{Material Safety Data Sheets (MSDS)}

In the U.S., the Occupational Safety and Health Administration (OSHA) establishes the rules that govern safety and health issues in the work place. Their regulation "1910.120 Hazardous waste operations and emergency response" (OSHA 1995) is the basis for Material Safety Data Sheets (MSDS) and is detailed in section 1910.1200-Hazard Communication (OSHA 1996a). (The following comments are meant only to be introductory in nature and it is recommended that interested readers download their own copies of these regulations through the internet addresses listed in the References.) Examples of MSDS for a wide range of chemicals are readily available through the internet. (MSDS 1986)

These informational data sheets are the responsibility of the manufacturer to provide to their employees and to those purchasing their products. The basic principle is that people have a right to know what the risks are in the work place and in the use of the products. This places the burden on the employers to know the risks involved with their work place and products. OSHA does not have a strict guideline as to how to determine these risks, but the employers have to demonstrate that they have adequately done so. The employers must communicate the risks involved with normal operations, as well as unplanned, but foreseeable, events such as equipment failure, containers rupturing, or control equipment malfunctions. Health hazards which must be communicated include chronic or acute problems with the material being carcinogenic, toxic, irritating, corrosive, sensitizing, reproductive toxins, etc., or which may cause damage to the lungs, skin, eyes, etc. The primary routes to entry into the body are to be listed and what are the allowable OSHA PEL or ACGIH Threshold limits. It must be stated if the material has been found to be carcinogenic by NTP, IARC, or OSHA. The physical and chemical hazards of the material, e.g., vapor pressure, flash point, potential for fire, explosion, and reactivity must be included.

The ingredients of the products must be listed if they are hazardous as determined by OSHA in 29 CFR part 1910, subpart Z (OSHA 1993) or by the American Conference of Govemmental Industrial Hygienists' "Threshold Limit Values for Chemical Substances and Physical Agents in the Work Environment" (ACGIH 1996). To determine if the product is carcinogenic or potentially carcinogenic, the National Toxicology Program's "Annual Report on Carcinogens" (NTP 1996a), International Agency for Research on Cancer's "Monographs" (IARC 1996) or OSHA's 29 CFR part 1910, subpart Z (OSHA 1993) are to used as sources. The contents of these sources are summarized in the National Institute for Occupational Safety and Health (NIOSH) "Registry of Toxic Effects of Chemical Substances (RTECS 1993), which was used as 
a basis for Table 1. The OSHA, NTP, and IARC lists can be downloaded through the internet addresses listed in the References. The threshold values may be purchased on a CD-ROM (ACGIH 1996).

If a mixture has been tested as whole, the observed toxic hazards are to be those reported on the MSDS. In the case where the mixture has not been tested as whole, the toxicity of the ingredients over $1 \%$ must be listed (over $0.1 \%$ if the ingredient is carcinogenic or potentially carcinogenic). The toxicity of the mixture is considered to be the same as that of components. For example, the equivalent exposure to air contaminated by a mixture is the sum of the component concentrations divided by the PEL for that component, with the resultant equivalent exposure to be less than unity to be in compliance (OSHA 1993).

However, if an accidental release of the mixture would release the components in other concentrations so as to exceed the PEL or be present in harmful concentrations, then this must be taken into consideration. For example, venting vapors from a storage tank full of pyrolysis oil would release vapors having more volatiles than are present in the oil. (OSHA 1993)

In the present situation, where testing of whole fast pyrolysis oil for health effects has not been extensively performed and where the product from scaled up fast pyrolysis systems has not been tested, it would seem necessary to rely on both the whole oil test results and on the known hazards associated with the individual chemicals reported to be found in fast pyrolysis oil.

In discussions with a representative of the U.S. Environmental Protection Agency (EPA) concerning the use of a new fuel, it appeared that they were not at all concerned with the toxicity of the fuel itself. However, they were very concerned with how clean the fuel would burn and what pollutants would be exhausted to the atmosphere in the flue gases. The combustion emissions will need to meet local standards. (Caldwall 1996) It is felt that these aspects are outside of the scope of this report, as they will be a complex function of the combustion equipment employed and how it is operated.

\section{Conclusions}

Wood smoke, wood distillates, and fast pyrolysis oils are not the same, but have a large number of oxygenated organic chemical compounds in common. The amount of aromatic hydrocarbons in these materials is related to the temperatures experienced in their production, with smoke having the potential for relatively high contents of monocyclic and polycyclic aromatic hydrocarbons compared to wood distillates and to properly made fast pyrolysis oils. There appear to be more aldehydes in wood smoke and fast pyrolysis oils than in wood distillates.

Aldehydes and unsaturated oxygenates pose the greatest acute toxic threat from fast pyrolysis oils, followed by furans. However, this toxicity may decrease with time as these compounds react with themselves and the many components in the fast pyrolysis oil. The projected toxicity of the known compounds at their reported concentrations in fast pyrolysis oils suggest only moderate acute oral toxicity of around $700 \mathrm{mg} / \mathrm{kg}$. The toxicity of fast pyrolysis oils to skin is low, but the 
eyes are very sensitive to this material. Exposure to aerosols of pyrolysis oil (smoke) is very irritating to the respiratory tract, can cause eye irritation and damage, and even death at high exposure levels over prolonged periods (however, because of the immediate eye, nose, and throat irritation, personnel will not voluntarily expose themselves to prolonged high levels of exposure to smoke). Most aerosol or smoke exposure will occur during production of the oil, with carbon monoxide gas probably the most toxic compound present in any escaping smoke.

Although $\beta$-propiolactone has not been reported in fast pyrolysis oils, wood smoke, nor wood distillation, its toxicity warrants a search for its presence. Due to the complexity of fast pyrolysis oils, its presence may have been overlooked. Certainly the presence of a small peak at M/Z 72 in the spectra of pyrolysis vapors made at $500^{\circ} \mathrm{C}$ (Evans and Milne 1987) indicates the possibility of this compound, although there are other possible compounds to explain this peak, e.g., propenic acid or pentane. However, since this lactone is very chemically reactive, it may not survive long in the complex organic mixture. As stated in the OSHA regulations, it is the demonstrated toxicity of the mixture that is more important, rather than the toxicity of the components.

The chronic toxicity of fast pyrolysis oils is unclear with one study indicating no tumor promoting ability and other studies showing mutagenicity or chromosome damage. However, the chronic exposure of humans to wood smoke did not result in an increase in the occurrence of lung cancer. The amount of $\mathrm{PAH}$ and $\mathrm{BaP}$ reported in fast pyrolysis oils is encouragingly very small, but the presence of formaldehyde and acetaldehyde could cause it to have some dangerous long term effects. This needs to be further evaluated to determine how long these reactive aldehydes are actually present in the reactive mixture of compounds in fast pyrolysis oils to pose health problems.

The toxicity of fast pyrolysis oils will be a function of composition, which in turn is process and feedstock dependent, and possibly with age. Consequently, extrapolation of the reported toxicity to other fast pyrolysis oils made with other systems may result in erroneous conclusions. Although this would appear to require a lot of testing of fast pyrolysis oils, it should be pointed out that the petroleum derived fuel oil now in use is not well characterized, varies in composition from refinery to refinery, and is suspected of carcinogenicity.

An MSDS is needed, using the best available information. It should be updated periodically and eventually have data in it generated with the specific pyrolysis system and feedstocks. This suggests that a fraction of the R\&D budget be allocated annually to further toxicology studies.

\section{Recommendations}

Although Elliott (1986 and 1988) showed no tumorgenicity with a fraction of fast pyrolysis oil made at NREL, due to the mutagenic results recently obtained with whole pyrolysis oils (Gratson 1994), it would seem prudent to repeat the chronic dermal animal tests both as tumor initiators and as promoters with a whole oil recently made in a scaled up pyrolysis system. After routine, daily production of fast pyrolysis oil is achieved, it would be of interest to test bi-weekly dermal 
applications to test animals of freshly made pyrolysis oils having less than one hour of age as tumor initiators and promoters to simulate possible operator exposure in the pyrolysis plant.

Although, some fractions of pyrolysis oils are used as food flavors, the oral toxicity of a fresh whole oil should be established, rather than just relying on the toxicity of the known components. Because there is only a small fraction of the oil that is actually identified chemically, there is a chance that there are some unknown toxic compounds present.

Air sampling needs to be done for formaldehyde and acrolein during the production of fast pyrolysis oils in the work space. It is recommended that the standard OSHA test for these compounds be used (OSHA 1996c). These compounds are reactive and volatile, so their contribution to the toxicity of the atmosphere of the operating environment may be underestimated, if only the pyrolysis oil is analyzed.

The use of organic vapor masks to reduce the exposure of personnel to fugitive fast pyrolysis oil vapors is recommended, but with the caveat that there may be dangerous levels of carbon monoxide also present where there are strong smoke odors in the pyrolysis plant. Small handheld carbon monoxide detectors are commercially available and are strongly recommended to be used by the operators, because the olfactory senses can become overloaded and the operators could lose the ability to smell the smoke odors after chronic exposure. These carbon monoxide detectors are also very useful to locate small gas leaks in the pyrolysis system. In instances where the operator is exposed to high levels of carbon monoxide or pyrolysis gases, air-supplied face masks are clearly needed. In addition, the use of carbon monoxide dosimeters may be useful to monitor accumulative exposure of the operators to pyrolysis gases, similar to those in use by forest firefighters.

Fast pyrolysis oils are chemically active and slowly react to form higher molecular weight compounds during aging. At this time, it is not known what the composition is of any gases that may be produced during this aging process. It is recommended that the composition of the vapor space above the pyrolysis oil be monitored during aging at various temperatures, with particular emphasis on looking for changes in carbon monoxide, formaldehyde, acetaldehyde, and acrolein concentrations. The composition of gases generated during storage will influence the methods permissible to vent off-gases to the atmosphere during transfer of pyrolysis oils from one tank to another.

Other toxicity problems associated with the production of fast pyrolysis oils include the dermal and inhalation toxicity to mammals that may associated with fine char dust, suggested by the chronic toxicity of solid coal pitch and carbon black. The toxicity of the char fines will probably depend upon whether they were exposed to a high temperature environment (e.g., partial combustion), so this will be very dependent upon the pyrolysis system sampled. Carbon black is listed as possibly carcinogenic to humans. In addition, respirable ceramic fibers are reasonably anticipated to be carcinogenic and respirable glass wool fibers are possibly carcinogenic to humans. (IARC 1996) Both types of fibers are used in thermal insulation materials. Wood dust has been recently reported by the International Agency for Research of Cancer to be carcinogenic, similar to formaldehyde (IARC 1995) Proper protection for operators in the feedstock 
preparation, char handling area, and around fibrous insulation must be taken to minimize these inhalation exposures.

Many possible scenarios exist for the deployment of fast pyrolysis facilities, which include the transport of the pyrolysis oil some distance from where it was produced to the site of the user. During transport, the possibility exists for an accidental spill of some magnitude onto the ground and into the ground water. The toxicity to aquatic species and the biodegradability of fast pyrolysis oils in water and in soil should be investigated to predict the potential environmental damage and subsequent remediation that would be required in such an accident. This information would also be useful to design proper treatment for incidental waste water coming from the fast pyrolysis plant, e.g., size of holding ponds, dilution of effluents, etc.

It is recommended that an expert consultant in toxicity testing and MSDS requirements be retained to review this report and to direct future testing, as appropriate within the scope of anticipated scale-up efforts of fast pyrolysis oils. Because the chemical constituents in fast pyrolysis oils are known to be sensitive to the processing conditions and to the feedstock, toxicity tests should be conducted with oils produced in the scaled-up equipment. As more people become involved with these oils, more depth of knowledge is needed of the toxicity of the oils made in near commercial conditions.

It is recommended that a fraction of the annual R\&D budget associated with fast pyrolysis oils be allocated to the health and safety issues of producing and utilizing these clean buming fuels.

\section{References}

ACGIH (1996) TLVs ${ }^{\odot}$ And Other Occupational Exposure Values-1996, CD-ROM, American Conference of Governmental Industrial Hygienists, (down load purchase information at http://www.acgih.org/cgi-bin/pubshow.html)

Ames, B.N.; McCann, J.; and Yamasaki, E. (1975) "Methods for Detecting Carcinogens and Mutagens with the Salmonella/mammalian-microsome Mutagenicity Test," Mutation Research, 31, 347-364. (in Elliott 1986)

Anon. (1956) "Fireless Smokehouse Smoker," Food Engineering, 28, (6), pp. 65 and 151.

Asita, A.O.; Matsui, M. Nohmi, T.; Matsuoka, A.: Hayashi, M.; Ishidate, M., Jr.; Sofuni, T.; Koyano, M.; and Matsushita, H. (1991) 'Mutagenicity of Wood Smoke Condensates in the Salmonella/Microsome Assay," Mutat. Res. (NNA), 264, No. 1, pp7-14. (Medline Abstract)

Benson, S. (1992) “Grill Crazy," Health, July/August, pp. 20-22.

Boukis, I. (1996) "Practical Implications During Operation of a CFB Air-Blown Pyrolyser" BioOil Production and Utilisation. Proceedings of the $2^{\text {nd }}$ EU-Canada Workshop on Thermal 
Biomass Processing, A.V. Bridgwater and E.N. Hogan, eds., CPL Press, Newbury, U.K., pp.49-65.

Brocksiepe, H-G (1986) "Charcoal," Ullmann's Encyclopedia of Industrial Chemistry, $5^{\text {th }}$ Edition, Volume A6, W. Gerhartz, Y.S. Yamamoto, F.T. Campbell, R. Pfefferkorn, and J.F. Rounsaville, eds., $\mathrm{VCH}, \mathrm{pp} .157-162$.

Brown, D.B. (1996) "Continuous Ablative Regenerator System," Bio-Oil Production and Utilisation. Proceedings of the $2^{\text {nd }}$ EU-Canada Workshop on Thermal Biomass Processing, A.V. Bridgwater and E.N. Hogan, eds., CPL Press, Newbury, U.K., pp. 96-101.

Brown, D.B. and Black, J. (1992) "Method and Apparatus for Ablative Heat Transfer," International Patent Application WO 92/09671.

Caldwall, J. (1996) U.S. Environmental Protection Agency, Washington, D.C. Personal telephone conversation with the author on July 22 .

Cuevas, A.; Rebollo, L.; Reinoso, C.; Scott, D.S. (1996) "Union Fenosa Fast Pyrolysis Developments," Proceedings of the $2^{\text {nd }}$ EU-Canada Workshop on Thermal Biomass Processing, A.V. Bridgwater and E.N. Hogan, eds., CPL Press, Newbury, U.K., pp.82-85.

Diebold, J.P. (1984) private communication to D.C. Elliott at Pacific Northwest Laboratories, August 1.

Diebold, J.P. and Scahill, J.W. (1996) "Improvements in the Vortex Reactor Design," Proceeding of Developments in Biomass Thermochemical Conversion, Banff, Canada, May 20-24, Chapman and Hill, London, in press.

Doerr, R.C.; Wasserman, A.E.; and Fiddler, W. (1966) "Composition of Hickory Sawdust Smoke Low-Boiling Constituents," J. Agri. Food Chem., 14, No. 6, 662-665.

Elliot, D.C. (1986) Analysis and Composition of Biomass Pyrolysis/Gasification CondensatesFinal Report, Pacific Northwest Laboratory, Richland, WA, PNL-5943/UC-61D.

Elliott, D.C. (1988) "Relation of Reaction Time and Temperature to Chemical Composition of Pyrolysis Oils," Pyrolysis Oils from Biomass, E.J. Soltes and T.A. Milne, eds., ACS Symposium Series 376, pp. 55-65.

Evans, R.J. and Milne, T.A. (1987) "Molecular Characterization of the Pyrolysis of Biomass 1. Fundamentals," Energy and Fuels, 1, No. 2, pp. 123-138.

Fitko, R.; Markiewicz, K.; Markiewicz, H.; Szuperski, T.; and Krzymowska, H. (1977a) "The Zootechnical indexes and pathological changes in Animals after Smoke Flavoring Application in Subacute Toxicity Testing," Acta. Aliment. Pol., 3, p. 269. (In Maga 1988) 
Fitko, R.; Grabowski, J.; Krzymowska, H.; Mrkiewicz, K,; ad Szuperski, T. (1977b) “The Acute Toxicity of Smoke Flavoring," Acta. Aliment. Pol., 3, p. 275. (in Maga 1988)

Fraps, G.S. (1901) “The Composition of a Wood Oil," American Chemical Journal, 25, 26-53.

Fritz, W. (1974) "Formation of Carcinogenic Hydrocarbons During Thermal Treatment of Foods. VI. Investigation of the Contamination of Cereals Resulting from Smoke Drying," Nahrung, 18, 83.

Goos, A.W. and Reiter, A.A. (1946) "New Products from Wood Carbonization," Ind. Eng. Chem., 38, 2, 132-135.

Graham, R. G.; Freel, B.A.; and Huffnan, D.R. (1994) "Applications of Rapid Thermal Processing of Biomass," Advances in Thermochemical Biomass Conversion, A.V. Bridgwater, ed., Blackie Academic \& Professional, pp. 1275-1288.

Gratson, D. (1994) "Results of Toxicological Testing of Whole Oils Derived from the Fast Pyrolysis of Biomass, " Proceedings Biomass Pyrolysis Oil Properties and Combustion Meeting," T.A. Milne, ed., Estes Park, CO, Sept. 26-28, National Renewable Energy Laboratory, Golden, CO, NREL-CP-430-7215, pp.203-211.

Guillén, M.D.; Manzanos, M.J.; and Zabala, L. (1995) "Study of a Commercial Liquid Smoke Flavoring by Means of Gas Chromatography/Mass Spectrometry and Fourier Transform Infrared Spectroscopy," J. Agric. Food Chem., 43, 463-468.

Guillén, M.D. and Ibargoitia, M.L. (1996) "Relationships Between the Maximum Temperature Reached in the Smoke Generation Processes from Vitus vinifera L. Shoot Sawdust and Composition of the Aqueous Smoke Flavoring Preparations Obtained," J. Agri. Food Chem., 44, 1302-1307.

Hawley, L.F. (1923) Wood Distillation, The Chemical Catalog Company, Inc., N.Y.

Husaini, S.A. and Cooper, G.E. (1957) "Fractionation of wood Smoke and the Comparison of Chemical Composition of Sawdust and Friction Smoke," Food Technology, 11, No. 10, pp. 499-502.

IARC (1995) Wood Dust and Formaldehyde, Vol. 62 of the Monograph Series on the Evaluation of Carcinogenic Risks to Humans, International Agency for Research on Cancer, World Health Org., Lyons, France, 405 pp. (Abstract can be down loaded from http://www.iarc.fr/publications/vol62.htm)

IARC (1996) "IARC Monographs on the Evaluation of Carcinogenic Risks to Humans and their Supplements: A Complete List," International Agency for Research on Cancer, World Health Org., May 20, (List may be down loaded from http://www.iarc.fr/monoeval/crthall.htm) 
Kirk and Othmer (1983) "Tar and Pitch--Health and Safety Factors," Encyclopedia of Chemical Technology, Vol. 22, $3^{\text {rd }}$ ed., John Wiley and Sons, NY, pp. 591-592.

Kirk and Othmer (1991) "Acrolein and its Derivatives," Encyclopedia of Chemical Technology. Vol. 1, $4^{\text {th }}$ ed., John Wiley and Sons, N.Y. pp. 232-251.

Klar, M. (1925) The Technology of Wood Distillation, Chapman \& Hall, London (translated to English by Alexander Rule)

Kovac, R.J. and O'Neil, D.J. (1989) "The Georgia Tech Entrained Flow Pyrolysis Process," Pyrolysis and Gasification, G.L. Ferrer, K. Maniatis, A. Buekens, and A.V. Bridgwater, eds., Elsevier Applied Science, London, pp. 169-179.

Kozlowski, Z.P. (1969) "Investigations on the Chemical, Toxicological and Biological Properties of the Polish Smoke Extract," Proc. Eur. Meet. Meat Res. Workers, 15, p. 516. (in Maga 1988)

Kurttio, P.; Kalliokoski, P.; Lampelo, S.; and Jantunen, M.J. (1990) "Mutagenic Compounds in Wood-Chip Drying Fumes," Mutation Research, 242, 9-15.

Larsson, B.K.; Sahlberg, G.P., Eriksson, A.T.; and Busk, L.A. (1983) 'Polycyclic Aromatic Hydrocarbons in Grilled Foods," J. Agric. Food Chem, 32, 867. (in Maga 1988)

Liang, C.K.; Quan, N.Y.; Cao, S.R.; He, X.Z.; and Ma, F. (1988) "Natural Inhalation Exposure to Coal Smoke and Wood Smoke Induces Lung Cancer in Mice and Rats," Biomed. Environ. Sci., 1 (1), pp. 42-50. (Medline Abstract)

Lijinsky, W. and Shubik, P. (1964) "Benzo(a)pyrene and Other Polynuclear Hydrocarbons in Charcoal-Broiled Meat," Science, 145, 53-55.

Lijinsky, W. and Shubik, P. (1965) "The Detection of Polycyclic Aromatic Hydrocarbons in Liquid Smoke and Some Foods," Toxicology and Appl. Pharmacology, 7, 337-343.

Maga, J.A. (1987) "The Flavor Chemistry of Wood Smoke," Food Reviews International, $3(1 \& 2), 139-183$.

Maga, J.A. (1988) Smoke in Food Processing, CRC Press, Boca Raton, FL, 160pp.

Mezerette, C. and Girard, P. (1991) "Environmental Aspects of Gaseous Emissions from Wood Carbonisation and Pyrolysis Processes," Biomass Pyrolysis Liquids. Upgrading and Utilization, A.V. Bridgwater and G. Grassi, eds., Elsevier Applied Science, London, pp. 263-288. 
Milne, T.A.; Agblevor, F.; Davis, M.; Deutch, S.; and Johnson, D. (1996) "A Review of the Chemical Composition of Fast-Pyrolysis Oils," Proceedings of Developments in Thermochemical Conversion of Biomass, A.V. Bridgwater, ed., Banff, Canada, May 20-24, Chapman \& Hall, London.

MSDS (1986) Material Safety Data Sheets, compiled by the University of Utah, (down load from gopher://gopher.chem.utah.edu/)

Mumford, J.L.; He, X.Z.; Chapman, R.S.; Cao, S.R.; Harris, D.B.; Li, X.M.; Jiang, W.Z.; Xu, C.W.; Chuang, J.C.; Wilson, W.E.; and Cooke, M. (1987) "Lung Cancer and Indoor Air Pollution in Xuan Wei, China," Science, 235, 217-220.

Nelson, W.G. (1930) "Waste-wood Utilization by the Badger-Stafford Process," Ind. Eng. Chem., 22, No. 4, 312-315.

NTP (1996a) "Known Carcinogens, $7^{\text {th }}$ Annual Report on Carcinogens," National Toxicology Program, (can be down loaded from http://ntp-db.niehs.nih.gov/htdocs/ARC/knowncarcinogen_list.html)

NTP (1996b) "Reasonably Anticipated to be Carcinogen: Beta-Propiolactone (CAS No. 57-578), (can be down loaded from http://ntp-db.niehs.nih.gov/htdocs/ARC/ARC_RAC/Betapropiolactone.html)

OSHA (1993) "Toxic and Hazardous Substances," 1910. Subpart Z, U.S. Occupational Safety and Health Administration, June 30 (can be down loaded from http://www.oshaslc.gov/OshStd_toc/OSHA_Std_toc_1910.html)

OSHA (1995) “ Hazardous Waste Operations and Emergency Response," 20 CFR Ch. XVII (71-95 edition) 1910.120, U.S. Occupational Safety and Health Administration pp. 376-418.

OSHA (1996a) "Hazard Communication." 20 CFR Ch. XVII (7-1-95 edition) 1910.1200, U.S. Occupational Safety and Health Administration (can be down loaded from http://www.oshaslc.gov/OshStd_data/1910.1200.html)

OSHA (1996b) "13 Carcinogens (4-Nitrobiphenyl, etc.)", 20 CFR Ch. XVII (7-1-95 edition) 1910.1003, U.S. Occupational Safety and Health Administration, (can be down loaded from http://www.osha-slc.gov/OshStd_data/1910.1200.html)

OSHA (1996c) "Sampling Strategy and Analytical Methods for Formaldehyde," 1910.1048 App. B, U.S. Occupational Safety and Health Administration. (can be down loaded from http://www.osha-slc.gov/OshStd_data/1910.1048_App.B.html)

Pakdel, H. and Roy, C. (1991) "Hydrocarbon Content of Liquid Products and Tar from Pyrolysis and Gasification of Wood," Energy and Fuels, 5, pp. 427-436. 
Palmer, R.C. (1915) "Preliminary Experiments on the Effect of Temperature Control on the Yield of Products in the Destructive Distillation of Hardwood," J. Ind. Eng. Chem., 7, No. $8,663-669$.

Peacocke, G.V.C. and Bridgwater, A.V. (1996) "Ablative Fast Pyrolysis of Biomass for Liquids, Results, and Analyses," Bio-Oil Production and Utilization. Proceedings of the $2^{\text {nd }}$ EUCanada Workshop on Thermal Biomass Processing, A.V. Bridgwater and E.N. Hogan, eds., CPL Press, Newbury, UK, pp. 35-48.

Pierson, W.E.; Koenig, J.Q.; and Bardana, E.J., Jr. (1989) "Potential Adverse Health effects of Wood Smoke," West. J. Med., 151, No. 3, pp. 339-342. (Medline Abstract)

Porter, R.W.; Bratzler, L.J.; and Pearson, A.M. (1965) "Fractionation and Study of Compounds in Wood Smoke," J. Food Science, 30, 615-619.

Pszczola, D.E. (1995) Tour Highlights Production and Uses of Smoke-Based Flavors," Food Technology, January, 70-74.

Reed, T.B. (1988) "Contact Pyrolysis in a Pyrolysis Mill," Research in Thermochemical Biomass Conversion, A.V. Bridgwater and J.L. Kuester, eds., Elsevier Applied Science, London, pp. 192-202.

Reinhardt, T.; Hanneman, A.; and Ottmar, R. (1994) Smoke Exposure at Prescribed Burns, Global Environmental Protection Program, USDA Forest Service, Seattle Forestry Sciences Laboratory, Pacific Northwest Research Station, 4043 Roosevelt Way, Seattle, WA 98105. (abstract may be down loaded from http://www.fs.fed.us/land/smoke.1.htm)

Riha, W.E.; Wendorff, W.L.; and Rank, S. (1992) "Benzo(a)pyrene Content of Smoked and Smoke-Flavored Cheese Products Sold in Wisconsin," J. Food Protection, 55, No. 8, pp. 636-638.

Riha, W.E. and Wendorff, W.L. (1993) "Browning Potential of Liquid Smoke Solutions: Comparison of Two Methods," J. Food Sci., 58, No. 3, pp. 671-674.

Roy, C.; Lemieux, R.; de Caumia, B.; Blanchette, D. (1988) "Processing of Wood Chips in a Semicontinuous Multiple-Hearth Vacuum-Pyrolysis Reactor," Pyrolysis Oils from Biomass, E.J. Soltes and T.A. Milne, eds., ACS Symposium Series 376, pp. 16-30.

RTECS (1993) Registry of Toxic Effects of Chemical Substances, Sweet, D.V., ed., National Institute for Occupational Safety and Health, Cincinnati, OH. NIOSH Publication 93-1012.

Sax, N.I. (1979) Dangerous Properties of Industrial Materials, $5^{\text {th }}$ Edition, Van Nostrand Reinhold Co., NY, p. 342. 
Scott, D.S. and Piskorz, J. (1984) "The Continuous Flash Pyrolysis of Biomass," Can. J. Chem. E., 62, pp. 404-412.

Scott, D. (1987) Technical Evaluation of the Waterloo Fast Pyrolysis Process, Energy, Mines and Resources Canada, Ottawa, Ontario, Canada, DSS Contract File No: 20ST.31926-35026, ISBN 0-662-15614-5, pp. 104-123.

Shepard, R.J. (1982) The Risks of Passive Smoking, Oxford University Press, NY. (in Maga 1988)

Shichino, Y., Tatematsu, M.; Ohshima, H.; Barsch, H.; Furihata, C.; and Ito, N. (1992) "Effects of Hickory-Smoke on Development of Pepsinogen 1-altered Pyloric Glands in Rats," Food Chem. Toxicol., 30, No. 10, 859-64. (Medline Abstract)

Stamm, A.J. and Harris, E.E. (1953) Chemical Processing of Wood, Chemical Publishing Co., N.Y.

Sternitzke, A.; Legrum, W.; and Netter, K.J. (1992) "Effects of Phenolic Smoke Condensates and their Components on Hepatic Drug Metabolizing Systems," Food Chem. Toxicol., 30, No. 9, pp. 771-781. (Medline Abstract)

Tóth, L. and Potthast, K. (1984) "Chemical Aspects of the Smoking of Meat and Meat Products," Advances in Food Research, vol. 29, pp. 87-158.

Underwood, G.L. and Graham, R.G (1989) "Method of Using Fast Pyrolysis Liquids as Liquid Smoke," U.S. Patent 4,876,108. (quoted in Riha and Wendorff 1993)

Underwood, G. (1992) "Commercialization of Fast Pyrolysis Products," Biomass Thermal Processing, Proceedings of the First Canada/European Community R\&D Contractors Meeting, E. Hogan, J. Robert, G. Grassi, and A.V. Bridgwater, eds., Chameleon Press Limited, London, pp. 226-228.

Vergnet, A-M. and Villeneuve, F. (1988) "Techniques Analytiques Applicables Aux Gaz et Jus de Pyrolyse de la Biomasse Tropicale," Cahiers Scientifiques. Bois et Forets des Tropiques, No. 9, pp. 3-66.

White, R.H.; Howard, J.W.; and Barnes, C.J. (1971) "Determination of Polycyclic Aromatic Hydrocarbons in Liquid Smoke Flavors," J. Agri. Food Chem., 19, No. 1, 143-146.

Wickstrom, K.; Pyysalo, H.; Plaami-Heikkila, S.; and Tuominen, J. (1986) "Polycyclic Aromatic Compounds (PAC) in Leaf Lettuce," Z. Lebensm. Unters. Forsch., 183, 182. (in Maga 1988)

Williams, P.T. and Home, P.A. (1995) "Analysis of Aromatic Hydrocarbons in Pyrolysis Oil derived from Biomass," JAAP, 31, pp. 15-37. 
Wynder, E.L. and Hoffinann, D. (1968) "Expenimental Tobacco Carcinogenesis," Science, 162, 862-871. 


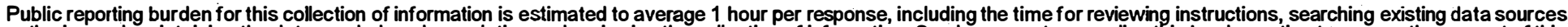

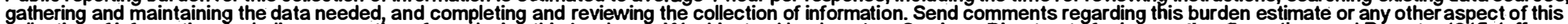

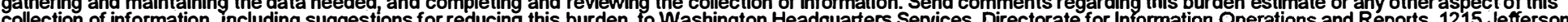

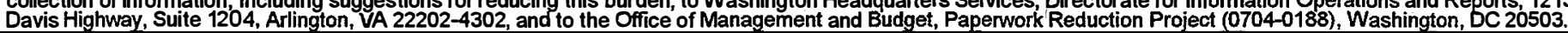
1. AGENCY USE ONLY (Leave blank)

\section{REPORT DATE}
3. REPORT TYPE AND DATES COVERED
April 1997
NREL Technical Report

4. TITLE AND SUBTITLE

A Review of the Toxicity of Biomass Pyrolysis Liquids Formed at Low Temperatures

5. FUNDING NUMBERS

Task\#: BP13432

\section{AUTHOR(S)}

J.P. Diebold

\section{PERFORMING ORGANIZATION NAME(S) AND ADDRESS(ES)}

Thermalchemie,Inc.

57 N. Yank Way

Lakewood, $\mathrm{CO}$

9. SPONSORING/MONITORING AGENCY NAME(S) AND ADDRESS(ES)

National Renewable Energy Laboratory

1617 Cole Boulevard

Golden, CO 80401-3393

8. PERFORMING ORGANIZATION REPORT NUMBER

DE97000231

10. SPONSORING/MONITORING AGENCY REPORT NUMBER

NRELTP-430-22739

\section{SUPPLEMENTARY NOTES}

\section{2a. DISTRIBUTION/AVAILABILITY STATEMENT}

National Technical Information Service

U.S. Department of Commerce

5285 Port Royal Road

Springfield, VA 22161 12b. DISTRIBUTION CODE

UC-600

13. ABSTRACT (Maximum 200 words) The scaleup of biomass fast pyrolysis systems to large pilot and commercial scales will expose an increasingly large number of personnel to potential health hazards, especially during the evaluation of the commercial use of the pyrolysis condensates. Although the concept of fast pyrolysis to optimize liquid products is relatively new, low-temperature pyrolysis processes have been used over tha aeons to produce charcoal and liquid by-products, e.g., smoky food flavors, food preservatives, and aerosols containign narcotics, e.g., nictotine. There are a number of studies in the historical literature that concern the hazards of acute and long-term exposure to smoke and to the historical pyrolysis liquids formed at low temperatures.
14. SUBJECT TERMS
pyrolysis, toxicity, benzene, polycyclic aromatic hydrocarbons
15. NUMBER OF PAGES 35

16. PRICE CODE

\section{SECURITY CLASSIFICATION} OF REPORT

\author{
18. SECURITY CLASSIFICATION \\ OF THIS PAGE
}

19. SECURITY CLASSIFICATION

OF ABSTRACT
20. LIMITATION OF ABSTRACT

Standard Form 298 (Rev. 2-89)

Prescribed by ANSI Std. Z39-18

298-102 